\title{
MAGNETAR BROADBAND X-RAY SPECTRA CORRELATED WITH MAGNETIC FIELDS: SUZAKU ARCHIVE OF SGRS AND AXPS COMBINED WITH NUSTAR, SWIFT, AND RXTE
}

\author{
Teruaki Enoto ${ }^{1,2}$, Shinpei Shibata $^{3}$, TAkao Kitaguchi $^{4}$, \\ Yudai Suma ${ }^{5}$, Takahiko Uchide ${ }^{6}$, Hiroyuki Nishioka ${ }^{7}$, Shota Kisaka ${ }^{8}$, \\ Toshio Nakano ${ }^{9}$, Hiroaki Murakami ${ }^{7}$, and Kazuo Makishima ${ }^{7,10}$ \\ temporal version
}

\begin{abstract}
Studies were made of the $1-70 \mathrm{keV}$ persistent spectra of fifteen magnetars as a complete sample observed with Suzaku from 2006 to 2013. Combined with early NuSTAR observations of four hard X-ray emitters, nine objects showed a hard power-law emission dominating at $\gtrsim 10 \mathrm{keV}$ with the 15-60 keV flux of $\sim 1-11 \times 10^{-11} \mathrm{ergs} \mathrm{s}^{-1} \mathrm{~cm}^{-2}$. The hard X-ray luminosity $L_{\mathrm{h}}$, relative to that of a soft-thermal surface radiation $L_{\mathrm{s}}$, tends to become higher toward younger and strongly magnetized objects. Updated from the previous study, their hardness ratio, defined as $\xi=L_{\mathrm{h}} / L_{\mathrm{s}}$, is correlated with the measured spin-down rate $\dot{P}$ as $\xi=0.62 \times\left(\dot{P} / 10^{-11} \mathrm{~s} \mathrm{~s}^{-1}\right)^{0.72}$, corresponding with positive and negative correlations of the dipole field strength $B_{\mathrm{d}}\left(\xi \propto B_{\mathrm{d}}^{1.41}\right)$ and the characteristic age $\tau_{\mathrm{c}}$ $\left(\xi \propto \tau_{\mathrm{c}}^{-0.68}\right)$, respectively. Among our sample, five transients were observed during X-ray outbursts, and the results are compared with their long-term 1-10 keV flux decays monitored with Swift/XRT and $R X T E / P C A$. Fading curves of three bright outbursts are approximated by an empirical formula used in the seismology, showing a $\sim 10-40 \mathrm{~d}$ plateau phase. Transients show the maximum luminosities of $L_{\mathrm{S}} \sim 10^{35} \mathrm{erg} \mathrm{s}^{-1}$, which is comparable to those of the persistently bright ones, and fade back to $\lesssim 10^{32} \mathrm{erg} \mathrm{s}^{-1}$. Spectral properties are discussed in a framework of the magnetar hypothesis.

Subject headings: catalog — pulsars: general — stars: magnetars — stars: magnetic field — stars: neutron - X-rays: stars
\end{abstract}

\section{INTRODUCTION}

Soft Gamma Repeaters (SGRs) and Anomalous X-ray Pulsars (AXPs) are growing to a new population of young neutron stars. These two subclasses were historically discovered in different ways (Kouveliotou et al. 1998; Mereghetti \& Stella 1995), but now are believed to have common observational properties (for recent reviews, see Woods \& Thompson 2006; Kaspi 2007; Mereghetti 2008); (a) a narrow range of slow spin periods, $P=2$ $12 \mathrm{~s}$, (b) high spin-down rates of $\dot{P}=10^{-12}-10^{-10} \mathrm{~s} \mathrm{~s}^{-1}$, (c) young characteristic ages as $\tau_{\mathrm{c}}=P / 2 \dot{P} \sim 1-100$ kyr (d) X-ray luminosities, $L_{\mathrm{x}} \sim 10^{34}-10^{35} \mathrm{erg} \mathrm{s}^{-1}$, that

\footnotetext{
1 The Hakubi Center for Advanced Research, Kyoto University, Kyoto 606-8302, Japan; teruaki_enoto@10.alumni.utokyo.ac.jp

2 Department of Astronomy, Kyoto University, KitashirakawaOiwake-cho, Sakyo-ku, Kyoto 606-8502, Japan

${ }^{3}$ Department of Physics, Yamagata University, Kojirakawa 14-12, Yamagata, 990-8560 Japan

${ }^{4}$ Department of Physical Science, Hiroshima University, 1-3-1 Kagamiyama, Higashi-Hiroshima, Hiroshima 739-8526, Japan

${ }^{5}$ Center for Gravitational Physics, Yukawa Institute for Theoretical Physics, Kyoto University, Oiwake-cho, Kitashirakawa, Sakyo-ku, Kyoto 606-8502, Japan

${ }^{6}$ Geological Survey of Japan, National Institute of Advanced Industrial Science and Technology (AIST), 1-1-1 Higashi, AIST Central 7, Tsukuba, Ibaraki 305-8567, Japan

${ }^{7}$ Department of Physics, University of Tokyo, 7-3-1 Hongo, Bunkyo-ku, Tokyo, 113-0033, Japan

${ }^{8}$ Department of Physics and Mathematics, Aoyama Gakuin University, 5-10-1 Fuchinobe, Sagamihara, Kanagawa 252-5258, Japan

${ }^{9}$ High Energy Astrophysics Laboratory, RIKEN Nishina Center, 2-1 Hirosawa, Wako, Saitama 351-0198, Japan

${ }^{10}$ MAXI team, Institute of Physical and Chemical Research (RIKEN), 2-1 Hirosawa, Wako, Saitama 351-0198
}

are brighter than the spin-down power $L_{\mathrm{sd}}=3.9 \times$ $10^{35} \mathrm{erg} \mathrm{s}^{-1} \dot{P}_{11} P_{1}^{-3} \sim 10^{32}-10^{34} \mathrm{erg} \mathrm{s}^{-1}$, where period and its derivative are normalized as $P_{1}=P /(1 \mathrm{~s})$ and $\dot{P}_{11}=\dot{P} /\left(10^{-11} \mathrm{~s} \mathrm{~s}^{-1}\right)$, respectively, (e) sporadic burst activities, and (f) occasional associations with supernova remnants.

The magnetar hypothesis for SGRs and AXPs (Duncan \& Thompson 1992; Thompson \& Duncan 1995, 1996) has become the most popular model in the last decade. This scenario describes that both SGRs and AXPs are isolated ultra-strongly magnetized neutron stars with their dipole magnetic field strength reaching $B_{\mathrm{d}}=1.0 \times 10^{14} \mathrm{G}\left(P_{1} \dot{P}_{11}\right)^{1 / 2} \sim 10^{14}-10^{15} \mathrm{G}$. In this model, high X-ray luminosity, $L_{\mathrm{x}} / L_{\mathrm{sd}} \sim 1-10^{3}$, is interpreted as a release of magnetic energies stored in the stellar interior. The "magnetar" model has come to be widely recognized as a fascinating concept, for example, in the context of supernova explosions (Nicholl et al. 2013), and in the extreme fundamental physics exceeding the quantum critical field $B_{\mathrm{QED}}=m_{\mathrm{e}}^{2} c^{3} /(\hbar e)=4.414 \times 10^{13} \mathrm{G}$ (Harding \& Lai 2006), where $m_{\mathrm{e}}, e, c$, and $\hbar$ are the electron rest mass, electron charge, speed of light, and Planck's constant, respectively.

Despite the accumulated evidence for the magnetar model, there are also alternative hypotheses proposed to explain the observational features of SGRs and AXPs in terms of, e.g., accretion from a fossil disk Alpar 2001a; Trümper et al. 2010), invoking a quark star model (Ouyed et al. 2004), or as fast rotating massive white dwarves (Malheiro et al. 2012). Therefore, it is imperative at this stage to observationally examine the radiation properties of this group of objects, and understand their 
radiation properties in a unified way.

Nearly 23 confirmed SGRs and AXPs are now known on the Galactic plane as displayed in Figure 1 with their timing properties in Figure 2 (see the detailed catalog, Olausen \& Kaspi 2014). Some are persistently bright with stable $L_{\mathrm{x}}$, intensively studied since the early days of X-ray astronomy: e.g., 4U 0142+61 (e.g., White et al. 1996; Enoto et al. 2011; Dib \& Kaspi 2014). The soft $\mathrm{X}$-ray spectrum is approximately an optically-thick radiation with its blackbody temperature at $k T \sim 0.5 \mathrm{keV}$ which is thought to originate from the stellar surface or vicinity as a quasi-thermal emission (Zane et al. 2009). In this paper, we call this soft radiation below $10 \mathrm{keV}$ "Soft X-ray Component (SXC)".

Recent observations have revealed a new distinctive "Hard X-ray Component (HXC)" dominating above 10 keV. First detected with INTEGRAL from persistently bright sources (Kuiper et al. 2006; den Hartog et al. 2008a, b) , the HXC was later reconfirmed by Suzaku and NuSTAR (Morii et al. 2010; Enoto et al. 2011; An et al. 2013a). This HXC extends up to $100 \mathrm{keV}$ or more with a hard photon index $\Gamma_{\mathrm{h}} \sim 1$, but must cuts off at some energies because of an upper limit by the $C G R O / C O M P T E L$ at $\gtrsim 1 \mathrm{MeV}$. This power-law HXC is now believed to be an optically thin emission presumably from a pulsar magnetosphere in the magnetar scheme (e.g., Beloborodov 2013a).

There are also subsequent discoveries of transient objects, mainly discovered by burst activities: e.g., SGR 0501+4516 (e.g., Enoto et al. 2009; Rea et al. 2009). Such transient sources occasionally cause surges in persistent X-rays by a few orders of magnitude, followed by a gradual decay (Rea \& Esposito 2011). During these "outburst" states, sporadic short bursts with short time-scale durations ( $\sim 1$ s) have been detected (Nakagawa et al. 2007; Israel et al. 2008). Although a complete picture has yet to come, bursts are thought to be originate from magnetic reconnection (Lvutikov 2003) or cracking of the crust with starquakes (Thompson et al. 2002).

The SXC and HXC match ideally with the simultaneous $0.2-600 \mathrm{keV}$ broadband coverage of the Suzaku satellite (Mitsuda et al. 2007). Our previous study of 9 SGRs and AXPs utilizing Suzaku (Enoto et al. 2010a, hereafter Paper I) suggested that 1) phase-averaged Xray radiation of SGRs/AXPs commonly consists of the SXC below $10 \mathrm{keV}$ and the HXC above $10 \mathrm{keV}$ in both quiescent states and transient outbursts, 2) $\Gamma_{\mathrm{h}}$ depends on $B_{\mathrm{d}}$ and $\tau_{\mathrm{ch}}$, and 3 ) their wide-band spectral properties are tightly correlated with $B_{\mathrm{d}}$ and $\tau_{\mathrm{ch}}$.

As the detailed description following Paper I, this paper provides a summary of Suzaku observations of SGRs and AXPs, combining the systematic spectral study of all the Suzaku sources and the X-ray decaying behavior of transient sources.

\section{OBSERVATION AND DATA REDUCTION}

\subsection{Suzaku Observations}

\subsubsection{Persistently bright or transient sources}

Table 1 summarizes all SGRs and AXPs which Suzaku has observed as of 2013 December. In this table, the "transient sources" exhibit prominent soft X-ray increases by $2-3$ orders of magnitudes and subsequent de- cays on timescales of months to years, while the "persistently bright ones" are relatively stable with their X-ray luminosities around $10^{35} \mathrm{ergs}^{-1}$. This is illustrated in Figure 3 as long-term X-ray flux records of representative sources. Since this "persistent" and "transient" classification is somehow phenomenological without a clear consensus (e.g., Pons \& Rea 2012a), we classified in this paper relatively variable sources as "transients".

Our Suzaku sample in Table 1 includes 16 pointings of 9 persistently bright sources and 10 target of opportunity ( $\mathrm{ToO}$ ) observations to follow-up 6 transient objects. This covers 15 objects of all the $\sim 29$ sources or candidates known to datt. Due to operational constraints, we were unable to observe recent transients SGR 0418+5729 and SGR 1745-29. In the following analyses, we reprocessed all the published data while adding newly observed objects, and performed comprehensive phase-averaged spectroscopic analyses.

\subsubsection{Reduction of broad-band Suzaku spectra}

We reprocessed the X-ray Imaging Spectrometer (XIS, 0.2-12 keV; Koyama et al. 2007) and the Hard X-ray Detector (HXD, 10-600 keV; Takahashi et al. 2007; Kokubun et al. 2007) data using FTOOLS "aepipeline" of the HEADAS version 6.14 or later with latest calibration database (CALDB) and the standard screening criteria. Only the full window, $1 / 4$, or $1 / 8$ window modes of XIS0, 1, and 3 (Table 1) are analyzed, since XIS2 has been out of operation due to damage by a micro meteorite in 2006 November. As for the HXD, only the HXD-PIN data were utilized since the typical SGR/AXP intensity in hard X-rays ( $\lesssim 1$ mCrab; Paper I) is below the detection limit of HXD-GSO.

The on-source and background XIS spectra were extracted from a circular region of $2^{\prime} .5$ radius and an annulus with the inner radius of $4^{\prime} .0$ and outer radius $7^{\prime} .5$ centered on the source, respectively. The XIS spectra of our magnetar sample are free from the pile-up effect, less than $\sim 1 \%$ (Yamada et al. 2012). The response matrix file (rmf) and auxiliary response file (arf) were produced using the FTOOLS xisrmfgen and xissimarfgen (Ishisaki et al. 2007). Two XIS0 and XIS3 spectra were summed up, with the rmf and arf also combined.

From the deadtime-corrected HXD-PIN spectrum of each source, we subtracted the Non X-ray Background (NXB), created with the LCFITDT method (Fukazawa et al. 2009), and filtered with the same criteria as those used in the observed on-source data. The Cosmic X-ray Background (CXB) was modeled as described by Enoto et al. 2010b) utilizing the refined spectral model as given by Moretti et al. (2009). Corresponding standard response files are used for this HXD-PIN spectrum. Thanks to the collimated field of view $\left(34^{\prime} \times 34^{\prime}\right.$ FWHM $)$, the spectra are free from source contamination except for three sources: CXOU J164710.2-45516 (a nearby bright X-ray source GX 340+0, Naik et al. 2008), AX J1818.8-1559 (a nearby hard source AX J1819.2-1601), and CXOU J171405.7-381031 (a potential contamination from a surrounding supernova remnant CTB $37 \mathrm{~B}$,

\footnotetext{
11 The latest magnetars and candidates are listed in http://www.physics.mcgill.ca/ pulsar/magnetar/main.html (see also., Olausen \& Kaspi 2014
} 


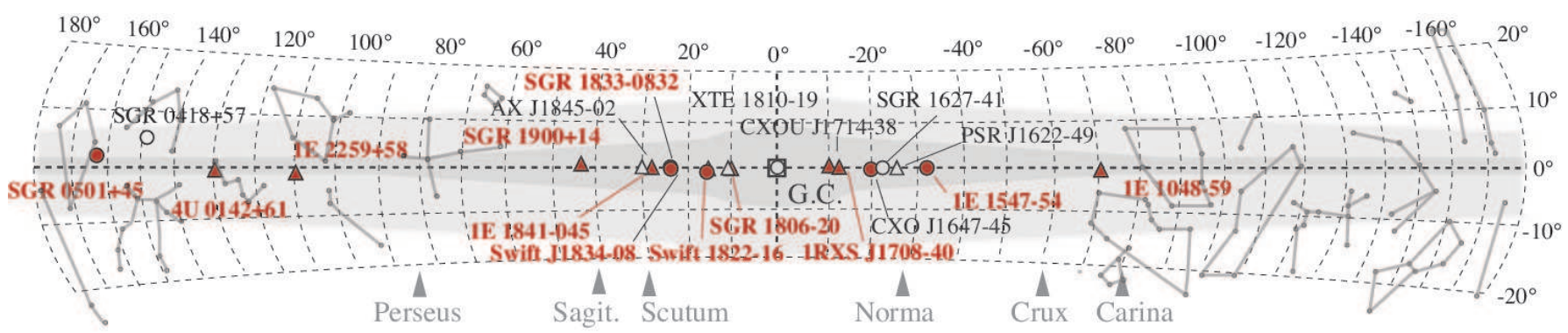

Figure 1. Known galactic SGRs and AXPs on the Galactic coordinate, where transient sources (circle) and persistently bright objects (triangle) are classified as in Table 1 The red filled symbols are sources observed with Suzaku. Spiral arms of our Galaxy are also indicated.

Table 1

Log of the Suzaku SGRs and AXPs observations.

\begin{tabular}{|c|c|c|c|c|c|c|c|c|}
\hline Name & $\begin{array}{c}B_{\mathrm{d}} \\
\left(10^{14} \mathrm{G}\right)\end{array}$ & ObsID & Start Time & Epoch & $\begin{array}{l}\text { Exp. } \\
(\mathrm{ks})\end{array}$ & $\begin{array}{l}\text { Nominal } \\
\text { Pointing }\end{array}$ & $\begin{array}{l}\text { XIS Mode } \\
\text { (XISO }, 1,3)\end{array}$ & $\begin{array}{l}\text { Process } \\
\text { Version }\end{array}$ \\
\hline \multicolumn{9}{|c|}{ Persistently Bright Sources } \\
\hline \multirow[t]{3}{*}{ SGR $1806-20$} & 24 & 401092010 & 2006-09-09 22:13:43 & $\mathrm{AO} 1$ & 48.9 & HXD & (full, full, full) & 2.0 .6 .13 \\
\hline & - & 401021010 & 2007-03-30 15:08:00 & $\mathrm{AO} 1$ & 19.6 & HXD & $(1 / 8,1 / 8,1 / 8)$ & 2.0 .6 .13 \\
\hline & - & 402094010 & 2007-10-14 05:35:49 & $\mathrm{AO} 2$ & 42.7 & HXD & (full, full, full) & 2.1 .6 .15 \\
\hline 1E $1841-045($ SNR Kes 73$)$ & 7.1 & 401100010 & 2006-04-19 10:51:40 & PV & 97.0 & HXD & $(1 / 8,1 / 8,1 / 8)$ & 2.0 .6 .13 \\
\hline \multirow[t]{2}{*}{ SGR $1900+14$} & 7.0 & 401022010 & 2006-04-01 08:42:57 & $\mathrm{AO} 1$ & 0.9 & HXD & $(1 / 4,1 / 8,1 / 4)$ & 2.0 .6 .13 \\
\hline & - & 404077010 & 2009-04-26 18:23:44 & Key & 40.6 & HXD & $(1 / 4,1 / 4,1 / 4)$ & 2.3 .12 .25 \\
\hline CXOU J171405.7-381031 & 5.0 & 501007010 & 2006-08-27 01:27:07 & $\mathrm{AO} 1$ & 75.6 & XIS & (full ,full ,full ) & 2.0 .6 .13 \\
\hline \multirow[t]{2}{*}{ 1RXS J170849.0-400910 } & 4.7 & 404080010 & 2009-08-23 16:25:08 & Key & 50.9 & HXD & $(1 / 4,1 / 4,1 / 4)$ & 2.4 .12 .26 \\
\hline & - & 405076010 & 2010-09-27 14:41:52 & Key & 47.0 & HXD & $(1 / 4,1 / 4,1 / 4)$ & 2.5.16.28 \\
\hline \multirow{5}{*}{$\begin{array}{l}1 \mathrm{E} 1048.1-593 \\
4 \mathrm{U} 0142+61\end{array}$} & 4.2 & 403005010 & 2008-11-30 23:02:01 & $\mathrm{AO} 3$ & 85.0 & HXD & (full, full, full) & 2.2 .11 .22 \\
\hline & 1.3 & 402013010 & 2007-08-13 04:04:13 & $\mathrm{AO} 2$ & 71.9 & HXD & $(1 / 4,1 / 4,1 / 4)$ & 2.1 .6 .15 \\
\hline & & 404079010 & 2009-08-12 01:41:15 & Key & 82.7 & HXD & $(1 / 4,1 / 4,1 / 4)$ & 2.4 .12 .26 \\
\hline & & 406031010 & 2011-09-07 15:43:32 & ToO & 36.7 & XIS & (full, full, 1/4) & 2.7 .16 .30 \\
\hline & & 408011010 & 2013-07-31 10:05:39 & $\mathrm{AO} 8$ & 79.8 & XIS & $(1 / 8,1 / 4,1 / 4)$ & 2.8 .20 .35 \\
\hline $1 \mathrm{E} 2259+586$ & 0.59 & 404076010 & 2009-05-25 20:00:17 & Key & 89.2 & HXD & $(1 / 4,1 / 4,1 / 4)$ & 2.4 .12 .26 \\
\hline AX J1818.8-1559 & & 406074010 & 2011-10-15 13:17:36 & $\mathrm{AO} 6$ & 88.5 & XIS & $(1 / 8,1 / 8,1 / 8)$ & 2.7 .16 .30 \\
\hline \multicolumn{9}{|c|}{ Transient Sources } \\
\hline \multirow[t]{2}{*}{$1 \mathrm{E} 1547.0-5408$} & 3.2 & 903006010 & $2009-01-2821: 34: 12$ & $\mathrm{ToO}$ & 10.6 & HXD & (psum, 1/4, 1/4) & 2.3 .12 .25 \\
\hline & - & 405024010 & 2010-08-07 03:52:07 & $\mathrm{AO} 5$ & 34.1 & HXD & $(1 / 4,1 / 4$, psum $)$ & 2.5.16.28 \\
\hline \multirow[t]{4}{*}{ SGR $0501+4516$} & 1.9 & 903002010 & 2008-08-26 00:24:42 & $\mathrm{ToO}$ & 43.3 & XIS & $(1 / 4,1 / 4,1 / 4)$ & 2.2 .8 .20 \\
\hline & - & 404078010 & 2009-08-17 20:21:51 & Key & 29.5 & HXD & $(1 / 4,1 / 4,1 / 4)$ & 2.4.12.26 \\
\hline & - & 405075010 & 2010-09-20 17:27:15 & Key & 49.1 & HXD & $(1 / 4,1 / 4,1 / 4)$ & 2.5.16.28 \\
\hline & - & 408013010 & 2013-08-31 23:25:40 & $\mathrm{AO} 8$ & 41.2 & XIS & $(1 / 8,1 / 4$, full $)$ & 2.8 .20 .35 \\
\hline SGR 1833-0832 & 1.8 & 904006010 & 2010-03-27 09:03:32 & $\mathrm{ToO}$ & 35.7 & HXD & $(1 / 8$, full, full $)$ & 2.5 .16 .28 \\
\hline CXOU J164710.2-455216 & 1.6 & 901002010 & 2006-09-23 06:59:17 & $\mathrm{ToO}$ & 38.7 & XIS & $(1 / 8,1 / 8,1 / 8)$ & 2.0 .6 .13 \\
\hline Swift J1834.9-0846 & 1.4 & 408015010 & 2013-10-17 07:17:57 & $\mathrm{AO} 8$ & 30.4 & XIS & (full, full, full) & 2.8 .20 .35 \\
\hline Swift J1822.3-1606 & 0.14 & 906002010 & 2011-09-13 09:59:07 & $\mathrm{ToO}$ & 36.1 & HXD & ( $1 / 8$, full, full $)$ & 2.7 .16 .30 \\
\hline
\end{tabular}

a Although there is accumulated evidence that SGRs and AXPs are intrinsically the same class of magnetars (Gavriil et al. 2002, Mereghetti 2008, Paper I), let us retain, in this paper, the historical terminology of "SGR" (usually discovered from burst activities) and "AXP" (identified as bright X-ray sources). Conventionally, some sources have a duplicated labeling both as the SGR and AXP; for example 1E 1547.0-5408 is also called SGR 1550-5418 or PSR J1550-5418. For such an object, we employ ether of the conventional names.

b Preceding Suzaku studies: SGR 1806-20 (Esposito et al. 2007; (Nakagawa et al. 2009), 1E 1841-45 (Morii et al. 2010), SGR 1900+14 (Nakagawa et al. 2009) 4U 0142+61 (Enoto et al. 2011; [Makishima et al. [2014), AX J1818.8-586 (Mereghetti et al. 2012), 1E 1547.0-5408 (Enoto et al. 2010b; [wahashi et al. 2013; Enoto et al. 2012; Makishima et al. 2015), SGR 0501+4516 (Enoto et al. 2009, 2010d; Nakagawa et al.|2011) CXOU J164710.2-455216 (Naik et al. 2008), SGR 1833-0832 (Esposito et al.|2011), Swift J1822.3-1606 (Rea et al. 2012b), and Paper I.

c Objects are sorted by the dipole field $B_{\mathrm{d}}$ calculated from the period $P$ and its derivative $\dot{P}$ assuming the magnetic dipole radiation. d Type of observations: PV (Performance Verification phase, i.e., first 9 month after the launch), AO (Announcement of Opportunity observations), ToO (Target of Opportunity observations), and Key (Key Project observations).

e Exposure time (ks) of one XIS instrument, the maximum value among the three cameras is shown.

${ }^{\mathrm{f}}$ Nominal pointing position (XIS or HXD) at the observations, see Mitsuda et al. (2007).

$g$ XIS Observation modes: The full window reads out every $8 \mathrm{~s}$, while $1 / 4$ and $1 / 8$ window modes read out every 2 and $1 \mathrm{~s}$, respectively. The timing P-sum mode (psum) provids a $\sim 7.8 \mathrm{~ms}$ time resolution together with one-dimentionally projected position information.

$\mathrm{g}$ Processing version of the data set. 


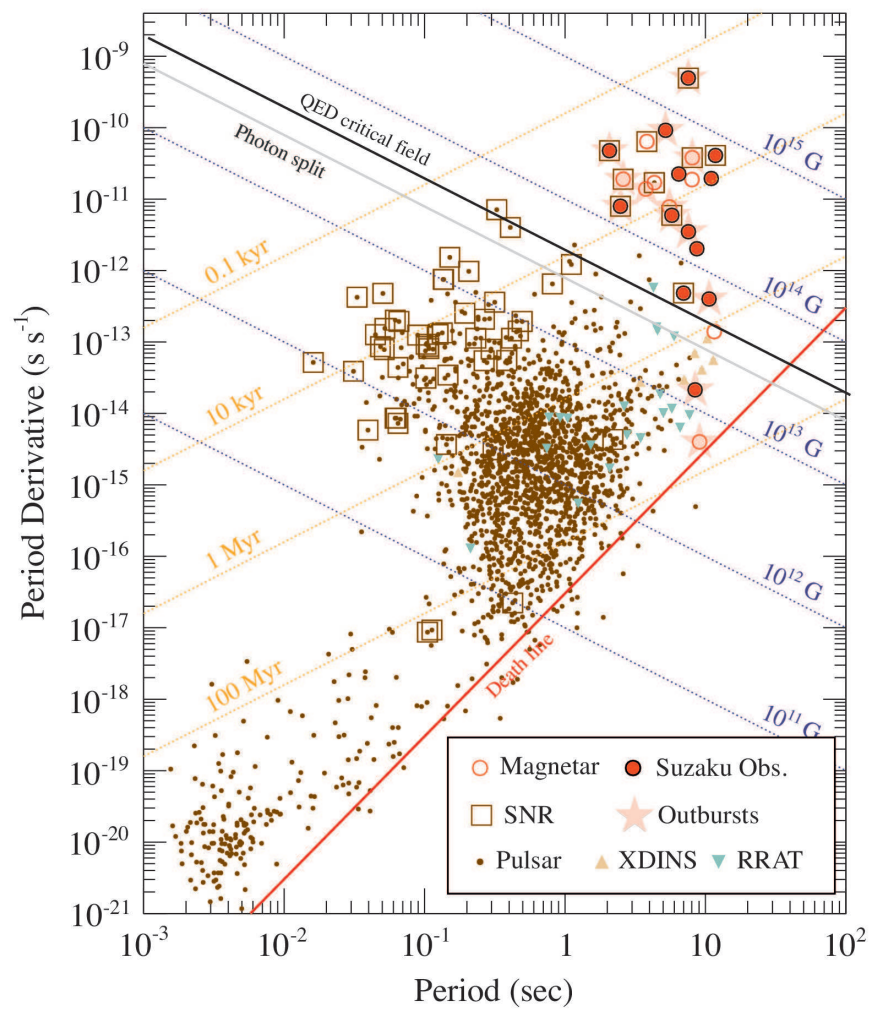

Figure 2. SGRs and AXPs on the $P-\dot{P}$ diagram, together with the grids of $B_{\mathrm{d}}, \tau_{\mathrm{c}}$, and $L_{\mathrm{sd}}$. The ATNF pulsar catalog is used for other pulsars (Manchester et al. 2005). Star and square symbols are objects showing X-ray outbursts and association with supernova remnants, respectively. The pulsar death death line (Chen \& Ruderman 1993; Zhang et al. 2000), B BED, and photon splitting line (Baring \& Harding 1998) are also indicated.

Nakamura et al. 2009).

The Galactic Ridge X-ray emission (GRXE; e.g., Krivonos et al. 2007) was further subtracted from the HXD-PIN spectrum when targets are close to the Galactic center[12]. We fixed the GRXE photon index at 2.1 (Valinia \& Marshall 1998) and employed normalization adjusted to reproduce near-by blank sky observations. The GRXE contribution is typically $\lesssim 3 \%$ of the NXB.

In the following analyses, all uncertainties quoted are given at the $68 \%(1 \sigma)$ confidence level for one parameter of interest unless stated otherwise.

\subsubsection{Detections of the soft and hard X-rays}

Figure 4 illustrates nine examples of the 1-10 keV XIS and $15-60 \mathrm{keV}$ HXD-PIN spectra derived by the procedures in 2.1.2. The SXC below $10 \mathrm{keV}$ was clearly detected with the XIS from all the observations in Table 1 except Swift J1834.9-0846 which had already been faded to become undetectable. Hereafter, we analyze the other sources.

After the NXB and CXB subtractions, we tabulate in Table 2 the 15-60 keV HXD-PIN source count rates $R_{\text {pin }}$

12 The GRXE are subtracted from SGR 1806-20, 1RXS J170849.0-400910, SGR 1833-0832, and 1E 1547.0-5408. For example, a blank-sky data (ObsID 500008010) was used for SGR 1806-20, while an observation of supernova remnant G25.5+0.0 (ObsID 504099010), which emits negligible signals in the HXD band, was utilized for SGR 1833-0832.
Table 2

HXD-PIN source rate and fluxes of the HXC.

\begin{tabular}{ccrrr}
\hline \hline Name & ObsID & $\begin{array}{r}T_{\text {pin }}^{\mathrm{a}} \\
(\mathrm{ks})\end{array}$ & \multicolumn{1}{c}{$R_{\text {pin }}^{\mathrm{b}}$} & \multicolumn{1}{c}{$F_{\mathrm{h}}^{\mathrm{c}}$} \\
\hline $1806-20$ & 401092010 & 51.9 & $6.0 \pm 0.3 \pm 0.3$ & $33.7 \pm 4.4$ \\
$1806-20$ & 401021010 & 15.6 & $5.8 \pm 0.5 \pm 0.5$ & $21.1 \pm 3.3$ \\
$1806-20$ & 402094010 & 46.6 & $4.7 \pm 0.3 \pm 0.3$ & $27.1 \pm 4.3$ \\
$1841-04$ & 401100010 & 59.8 & $6.7 \pm 0.3 \pm 0.3$ & $48.9 \pm 0.3$ \\
$1900+14$ & 401022010 & 13.5 & $3.4 \pm 0.6 \pm 0.6$ & $20.6 \pm 5.5$ \\
$1900+14$ & 404077010 & 39.1 & $3.5 \pm 0.3 \pm 0.3$ & $16.5 \pm 3.5$ \\
$1708-40$ & 404080010 & 47.9 & $4.0 \pm 0.3 \pm 0.3$ & $24.4 \pm 4.4$ \\
$1708-40$ & 405076010 & 55.4 & $4.5 \pm 0.3 \pm 0.3$ & $24.4 \pm 4.0$ \\
$1048-59$ & 403005010 & 63.3 & $<2.3$ & $<13.2$ \\
$0142+61$ & 402013010 & 94.7 & $4.1 \pm 0.2 \pm 0.2$ & $35.1 \pm 6.4$ \\
$0142+61$ & 404079010 & 92.5 & $3.1 \pm 0.2 \pm 0.2$ & $26.2 \pm 5.8$ \\
$0142+61$ & 406031010 & 39.3 & $4.7 \pm 0.3 \pm 0.3$ & $24.3 \pm 3.7$ \\
$0142+61$ & 408011010 & 96.2 & $3.8 \pm 0.2 \pm 0.2$ & $19.1 \pm 2.8$ \\
$2259+58$ & 404076010 & 96.0 & $<1.8$ & $<10.1$ \\
$1547-54$ & 903006010 & 31.0 & $17.4 \pm 0.4 \pm 0.4$ & $110.2 \pm 5.2$ \\
$1547-54$ & 405024010 & 39.6 & $3.2 \pm 0.3 \pm 0.3$ & $13.5 \pm 3.3$ \\
$0501+45$ & 903002010 & 50.7 & $3.0 \pm 0.3 \pm 0.3$ & $28.1 \pm 6.5$ \\
$0501+45$ & 404078010 & 25.5 & $<3.7$ & $<20.9$ \\
$0501+45$ & 405075010 & 48.9 & $<2.9$ & $<16.2$ \\
$0501+45$ & 408013010 & 33.4 & $<2.3$ & $<12.7$ \\
$1833-08$ & 904006010 & 10.0 & $3.9 \pm 0.7 \pm 0.7$ & $17.6 \pm 4.5$ \\
$1822-16$ & 906002010 & 33.7 & $<0.9$ & $<4.9$ \\
\hline
\end{tabular}

a $T_{\text {pin }}$ : The effective HXD exposure.

b The 15-60 keV HXD-PIN count rates with $1 \sigma$ statistical and systematic errors. If not detected, the $3 \sigma$ upper-limits are shown.

c The 15-60 keV absorbed flux in an unit of $10^{-12} \mathrm{ergs} \mathrm{s}^{-1} \mathrm{~cm}^{-2}$ with $1 \sigma$ statistical and systematic errors. If not detected, the $3 \sigma$ upper-limits are shown converted from the count rate $R_{\text {pin }}$ with a conversion factor of $F_{\mathrm{x}} / R_{\mathrm{pin}}=6 \times 10^{-12} \mathrm{erg} \mathrm{s}^{-1} \mathrm{~cm}^{-2} /\left(0.01 \mathrm{cnt} \mathrm{s}^{-1}\right)$.

d The Suzaku detected HXC above were also reported with other satellite: 4U 0142+61, 1RXS J170849.0-400910 (Kuiper et al.|2006; den Hartog et al. 2008b.a); 1E 1841-045 (An et al. 2013b); SGR 1806-20 SGR 1900+14 (Götz et al. 2006); 1E 1547.0-5408 (Kuiper et al. 2012); SGR 0501+4516 (Rea et al. 2009).

together with $1 \sigma$ statistical and systematic uncertainties. The systematic uncertainty of the HXD-PIN background is a sum of $1 \%$ level of the NXB (reproducibility of the LCFITDT model; Fukazawa et al. 2009) and 10\% of the CXB (1 $\sigma$ sky-to-sky fluctuation). The associated 15$60 \mathrm{keV}$ flux $F_{\mathrm{x}}$ was calculated via power-law fitting of the HXD data. If the HXC is undetectable, we set $3 \sigma$ upper-limits on the count rates, which were converted to those on $F_{\mathrm{x}}$ assuming spectral shapes of detected sources (caption of Table 2). We assigned $3 \sigma$ upper limits on 1E 2259+586, 1E 1048.1-537, Swift J1822.3-1606, and latter observations of SGR $0501+4516$. The upper limit on $1 \mathrm{E} 2259+586$ is consistent with a recent detection by NuSTAR (Vogel et al. 2014).

As reported in Paper I and references therein, we have detected the HXC from 7 sources with $>3 \sigma$ significance (see the caption in Table 1). Out of 10 new observations of 6 sources added to Paper I, the HXC was reconfirmed from 4U 0142+61 in 2011, 2013 and RXS J170849.0-400910 in 2010. A signature of the HXC was suggested in SGR 1833-0832, but $F_{\mathrm{h}}$ is rather weak compared with other sources, and the detection is marginal. Thus, we do not use this source in the correlation fittings in 83 . Figure 5 illustrates a comparison of $F_{\mathrm{s}}$ and $F_{\mathrm{h}}$. Suzaku has detected the HXC down to $\sim 10^{-11} \mathrm{ergs} \mathrm{cm}^{-2} \mathrm{~s}^{-1}$ in the $15-60 \mathrm{keV}$ band. 
Table 3

List of archived NuSTAR and Swift observations of SGRs and AXPs used in this work.

\begin{tabular}{|c|c|c|c|c|c|c|c|}
\hline \multirow[t]{2}{*}{ Name } & \multicolumn{3}{|c|}{ NuSTAR } & \multicolumn{3}{|c|}{ Swift/XRT } & \multirow[t]{2}{*}{ Ref. } \\
\hline & ObsID & Obs. Date & $(\mathrm{ks})$ & ObsID & Obs. Date & $(\mathrm{ks})$ & \\
\hline $1 \mathrm{E} 1841-045$ & $30001025[04,06,08,10,12]$ & 13 Sep 5-23 & 273 & 00080220004 & 13 Sep 21 & 1.8 & [1] \\
\hline $4 \mathrm{U} 0142+61$ & $300010230[02,03]$ & 14 March 27-30 & 168 & $000800260[01-03]$ & 14 March 27-30 & 24 & [2] \\
\hline $1 \mathrm{E} 2259+586$ & $300010260[02,03,05]$ & 13 April 24-27 & 157 & $000802920[02,03,04]$ & 13 April 25-28 & 30 & [3] \\
\hline 1E $2259+586$ & 30001026007 & 13 May $16-18$ & 88 & $000802920[05,07]$ & 13 May $16-18$ & 8.7 & \\
\hline
\end{tabular}

Note. - [1] An et al. (2013a); [2] Tendulkar et al. (2015) [3] Vogel et al. (2014)

\subsection{NuSTAR Observations}

The Nuclear Spectroscopic Telescope Array ( NuSTAR, Harrison et al. 2013) provides a high spectral sensitivity in the 3-79 keV band, and has already observed bright AXPs An et al. 2014b, 2013a, 2014a; Vogel et al. 2014; Tendulkar et al. 2015; Yang et al.|2015). In order to verify our results performed by the non-imaging instrument HXD, here we analyze initial $N u S T A R$ data sets available in the archive listed in Table 3 .

The data were processed and filtered with the standard nupipeline and nuproducts softwares of HEASOFT version 6.16 and the $N u S T A R$ CALDB version 20141020. The on-source spectra were extracted from a circular region of $1^{\prime} .0$ radius centered on the target objects within which nearly $90 \%$ photons are collected. Since some AXPs are faint hard X-ray sources, we used the background modeling software nuskybkg (Wik et al. 2014) for accurate background subtraction. This tool generates a simulated background spectrum expected on the selected source region by fitting blank-sky spectra from the same focal plane. We selected, for the background spectral modeling, three annual regions with radii $2^{\prime} .0-5^{\prime} .0,5^{\prime} .0$ $8^{\prime} .0$, and $8^{\prime} .0-12^{\prime} .3$ centered on the target sources for each telescope, and adjusted model parameters to explain the actual blank-sky data. The background spectrum was simulated from the best fit modeling parameters with a 100 times longer exposure to reduce statistical uncertainties. The accuracy of the simulated background spectrum was verified using blank sky data as described in Kitaguchi et al. (2014).

If there are simultaneous Swift coverage of during the NuSTAR observations, we also utilized the archived Swift/XRT data listed in Table 3 , after the data processing as described in 2.3 . If the observations were carried out in a sequential way during a month, we merged continuous data with different observation ID into one spectrum and response for our long-term and phase averaged analyses. Two series of observations of $1 \mathrm{E} 2259+586$ were performed in 2013 April and March, so we derived two spectra (Table 3). As an example, we show the background-subtracted X-ray spectra of $4 \mathrm{U} 0142+61$ in Figure 6] The derived NuSTAR spectra are consistent with previous works by An et al. 2013a, Vogel et al. 2014, and Tendulkar et al. 2015.

\subsection{Swift and RXTE Observations of Outbursts}

Transient magnetars are characterized by sporadic Xray outbursts, namely sudden increases of the persistent emission, which have recently provided a drastic increase of the number of this class (e.g., as early studies, Gavriil et al. 2002; Kouveliotou et al. 2003; Kaspi et al.
2003 and for a recent review, Rea \& Esposito 2011). The onset of an outburst is usually noticed by a detection of short bursts by the Swift Burst Alert Telescope (BAT; Gehrels et al. 2004; Barthelmy et al. 2005), and then monitored with the Swift X-ray Telescope (XRT; Burrows et al. 2005) or with Rossi X-Ray Timing Explorer ( $R X T E$; Bradt et al. 1993), as shown in Figure 7

We conducted Suzaku ToO observations of some transients usually within a week after. The obtained Suzaku snap shots are also presented in Figure 7. We studied characteristics of outbursts of latest known 8 outbursts of 7 sources which occurred after the Suzaku launch, as listed in Table 4 (see also Figure 3). The onset of an outburst is defined as the first short bursts reported by Swift/BAT or Frermi/Gamma-ray Burst Monitor (GBM).

We uniformly processed all the public available XRT data of 8 outbursts via the standard procedure FTOOLS xrtpipeline with default filtering criteria. We used the latest available RMF matrix in CALDB v20140610, while generated the ARF files with the xrtmkarf tool. For the imaging Photon Counting (PC) mode, we extracted source photons from a circular region with a $48^{\prime \prime}$ (20 pixels) radius centered on the target, while collected background spectra from annular regions with the inner and outer radii of $167^{\prime \prime}$ (70 pixels) and $286^{\prime \prime}$ (120 pixels), respectively. When the $\mathrm{PC}$-mode count rates exceed $\sim 0.5$ count $\mathrm{s}^{-1}$, we excluded a central 8.0" (3.4 pixels) region following a standard procedure 13 . For the Windowed Timing (WT) mode with an one-dimensional information and $1.76 \mathrm{~ms}$ time resolution, we extracted source and background spectra from a strip of $94^{\prime \prime}$ width around the source and surrounding regions by $140^{\prime \prime}$ away from the target, respectively. When the count rate exceeds $100 \mathrm{cnts}^{-1}$, we excluded the central 14"strip to avoid the pile-up. After the above standard pipelines, we discarded observations with poor photon statistics if the total source count is smaller than 100 cnts per observation.

The non-imaging $R X T E$ Proportional Counter Array (PCA; Jahoda et al. 2006) operates in the $2-60 \mathrm{keV}$ energy band with a full width at half-maximum field of view of $\sim 1^{\circ}$. Due to nearby sources, we only used the PCA data for SGR $0501+4516$ and SGR $0418+5729$. The data were processed via the standard procedure using FTOOLS rex, pcarsp, and recofmi tasks.

\section{ANALYSIS AND RESULTS}

\subsection{Spectral modeling of two X-ray components}

\footnotetext{
13 http://www.swift.ac.uk/analysis/xrt/pileup.php
} 
Table 4

List of X-ray outbursts from transient sources.

\begin{tabular}{|c|c|c|c|c|c|c|c|c|}
\hline Name & $\begin{array}{c}B_{\mathrm{d}} \\
\left(10^{14} \mathrm{G}\right)\end{array}$ & $\begin{array}{l}\text { Time Origin }^{\mathrm{a}} \\
\text { (UT) }\end{array}$ & Ref. & Model $^{\mathrm{b}}$ & $\begin{array}{c}L_{0} \mathrm{c} \\
\left(10^{34} \mathrm{erg} \mathrm{s}^{-1}\right)\end{array}$ & $\begin{array}{l}\tau_{0}{ }^{\mathrm{c}} \\
\text { (d) }\end{array}$ & $p^{\mathrm{c}}$ & $\begin{array}{l}E_{\mathrm{tot}}{ }^{\mathrm{c}} \\
(\mathrm{erg})\end{array}$ \\
\hline CXOUJ164710-455216 & 1.6 & 2006-09-21 01:34:52 & $1 a-d$ & PL & $8.2 \pm 0.3$ & - & $0.23 \pm 0.02$ & $3.1 \times 10^{41}$ \\
\hline SGR $0501+4516$ & 1.9 & $2008-08-22 \quad 12: 41: 59$ & $2 a-d$ & $\mathrm{PD}$ & $5.9 \pm 0.2$ & $15.9 \pm 2.9$ & $0.76 \pm 0.05$ & $1.5 \times 10^{43}$ \\
\hline 1E $1547.0-5408$ & 3.2 & 2008-10-03 09:28:08 & $3 a-d$ & PL & $9.1 \pm 0.4$ & - & $0.13 \pm 0.02$ & $4.8 \times 10^{41}$ \\
\hline 1E $1547.0-5408$ & 3.2 & 2009-01-22 01:32:41 & $4 \mathrm{a}-\mathrm{e}$ & PL & $25.7 \pm 0.6$ & - & $0.29 \pm 0.01$ & $7.9 \times 10^{41}$ \\
\hline SGR $0418+5729$ & 0.061 & 2009-06-05 20:40:48 & $5 \mathrm{a}-\mathrm{e}$ & PD & $6.1 \pm 0.4$ & $42.9 \pm 11.6$ & $1.99 \pm 0.30$ & $2.3 \times 10^{41}$ \\
\hline SGR $1833-0832$ & 1.8 & 2010-03-19 18:34:50 & $6 a-b$ & $\mathrm{PL}$ & $8.8 \pm 1.2$ & - & $0.07 \pm 0.06$ & $5.8 \times 10^{41}$ \\
\hline Swift J1822-16069 & 0.14 & 2011-07-14 12:47:47 & $7 \mathrm{a}-\mathrm{c}$ & $\mathrm{PD}$ & $7.2 \pm 0.3$ & $11.2 \pm 0.9$ & $1.25 \pm 0.02$ & $2.9 \times 10^{41}$ \\
\hline Swift J1834.9-0846 & 1.4 & 2011-08-07 19:57:46 & $8 \mathrm{a}-\mathrm{c}$ & PL & $1.8 \pm 0.4$ & - & $0.28 \pm 0.09$ & $5.7 \times 10^{40}$ \\
\hline
\end{tabular}

Note. - X-ray outbursts after the Suzaku and Swift launches, i.e., outbursts from 1E 2259+586 in 2002, XTE J1810-197 in 2003, and 1E 1048.1-5937 in 2007 are not included. Further details of individual outbursts are listed in Ref., (1a) Krimm et al. (2006); (1b) Israel et al. (2007); (1c) Naik et al. (2008); (1d) Clark et al. (2005); (2a) Holland et al. (2008); (2b) Enoto et al. (2009); (2c) Rea et al. (2009); (2d) Aptekar et al. (2009); (3a) Krimm et al. (2008); (3b) Israel et al. (2010); (3c) Tiengo et al. (2010); (4a) Gronwall et al. (2009); (4b) Enoto et al. (2010b); (4c) Ng et al. (2011); (5a) van der Horst et al. (2009); (5b) van der Horst et al. (2010); (5c) Rea et al. (2010); (5d) Esposito et al. (2010); (5e) Güver et al. (2011); (5d) Rea et al. (2013a); (6a) Gelbord et al. (2010); (6b) Göğüs et al. (2010); (6c) Esposito et al. (2011); (7a) Cummings et al. (2011); (7b) Rea et al. (2012b); (7c) Scholz et al. (2012); (8a) D'Elia et al. (2011); (8b) Kargaltsev et al. (2012); (8c) Esposito et al. (2013a);

a Time onset of the outbursts defined at the first short burst detected by Swift/BAT or Fermi/GBM. See the above references.

b Applied fitting model used to unabsorbed 2-10 keV X-ray light curves; A single power-law model $\left(\mathrm{PL}, L_{\mathrm{x}}(t)=L_{0}(t / 1 \mathrm{~d})^{-p}\right)$ and a plateau decaying function $\left(\mathrm{PD}, L_{\mathrm{x}}(t)=L_{0}\left(1+t / \tau_{0}\right)^{-p}\right)$.

c Best-fit parameters of unabsorbed $2-10 \mathrm{keV}$ X-ray light curves (see details in 93.3 ). $L_{0}$ is an initial X-ray luminosity at 1 day after the onset or during the plateau phase for the PL and PD models, respectively. $\tau_{0}$ and $p$ are a duration of the plateau and the slope, respectively. The total emitted energy $E_{\text {total }}$ is evaluated as an integration up to $100 \mathrm{~d}$ if $p<1$.

We carried out unified spectral fitting of the Suzaku phase-average broad-band spectra. Phase-resolved spectroscopy, available only for bright and slowly rotating objects (e.g., 1RXS J18049.0-400910), is beyond the scope of this paper. In order to avoid instrumental calibration uncertainties of the XIS, the 1.7-1.9 keV data were discarded, and a $2 \%$ systematic error was assigned to the XIS spectral bins. The XIS and HXD-PIN spectra were binned so that each bin has either $>5 \sigma$ significance or $>30$ counts. The cross-normalization factor of HXDPIN was fixed at 1.164 and 1.181 relative to XIS-FI in cases of the XIS- and HXD-nominal pointing positions (Maeda et al. 2008), respectively. The normalization of XIS-BI to XIS-FI was allowed to differ by up to $5 \%$. For the simultaneous Swift and NuSTAR spectra (\$2.2) the normalization was fixed to that of the Swift/XRT and the cross-normalization between the telescopes (FPMA and FPMB) was allowed by up to $5 \%$.

A photo-absorption factor was multiplied to an intrinsic continuum spectral model of the SXC. We employed the phabs model Balucinska-Church \& McCammon 1992) in XSPEC with the solar metallicity abundance angr (Anders \& Grevesse 1989) and cross-section bcmc; this combination has been widely used in the literature and allows us to compare with previous results. When the HXC is detected in the $15-60 \mathrm{keV}$ band, it was expressed by an additional single power-law. We further added a plasma emission model when analyzing the 1E 1841-045 and CXU J171405.74-381031 data, to describe the surrounding supernova remnant (SNR) Kes 73 (Kumar et al. 2014) and CTB 37B (Sato et al. 2010), respectively, as described in Appendix A.

The intrinsic SXC spectrum is conventionally fitted by a model comprising two blackbody components (hereafter 2BB model), or a combination of a blackbody plus an additional soft power-law (BB $+\mathrm{PL}$ model), The second high-energy component of both models is considered to represent either a temperature anisotropy over the stellar surface, an up-scattering of soft photons in the magnetosphere, or effects of a magnetised neutron star atmosphere (e.g., Lvutikov \& Gavriil 2006; Rea et al. 2008; Güver et al. 2011). These empirical 2BB and $\mathrm{BB}+\mathrm{PL}$ models roughly explain the data, though only approximately sometimes. The low and high blackbody temperatures in the $2 \mathrm{BB}$ model, $k T_{\mathrm{L}}$ and $k T_{\mathrm{H}}$, are known to follow a relation of $k T_{\mathrm{L}} / k T_{\mathrm{H}} \sim 0.4$ (Nakagawa et al. 2009), thus the number of free spectral parameters is expected to be three rather than four of the $2 \mathrm{BB}$ or $\mathrm{BB}+\mathrm{PL}$ models.

Since the SXC spectral modeling has not reached a consensus, we utilize an empirical blackbody shape with a Comptonization-like power-law tail (hereafter CBB model, Tiengo et al. 2005; Halpern et al. 2008; Enoto et al. 2010b, and Paper I). This CBB model is mathematically described by three parameters; the temperature $k T$, soft-tail power-law photon index $\Gamma_{\mathrm{s}}$, and normalization corresponding to the emission radius $R$, and the model reproduces the soft-tail at $\gtrsim 5 \mathrm{keV}$ of the $\mathrm{BB}+\mathrm{PL}$ model without a large $N_{\mathrm{H}}$ that is needed by the 2BB.

We present the Suzaku CBB best-fit parameters in Table 5 and corresponding $\nu F_{\nu}$ spectra in Figure 8 . The NuSTAR fit results are given in Table [5] together with some $\nu F_{\nu}$ examples in Figure 9. The NuSTAR $\nu F_{\nu}$ shapes of bright AXPs, $4 \mathrm{U} 0142+61$ and $1 \mathrm{E} 1841-045$, are consistent with those of Suzaku. The HXC of 1E 2259+586 was detected with $N u S T A R$ (Vogel et al. 2014), but not with Suzkau.

\subsection{Correlations among spectral parameters \\ 3.2.1. Ratio of HXC to SXC vs. magnetic field}

In Paper I, we proposed a broad-band spectral evolution of this class: i.e., i) the hardness ratio of the HXC to SXC is positively (or negatively) correlated to their $B_{\mathrm{d}}\left(\right.$ or $\tau_{\mathrm{c}}$ ), and ii) the HXC photon index $\Gamma_{\mathrm{h}}$ becomes harder toward the weaker $B_{\mathrm{d}}$ sources (see also 
Table 5

Best-fit parameters of SGR/AXP observations using the CBB+PL model with Suzaku and NuSTAR.

\begin{tabular}{|c|c|c|c|c|c|c|c|c|c|c|c|}
\hline Name & $\begin{array}{l}\text { ObsID } \\
\text { Month }\end{array}$ & $F_{1-10}$ & $F_{15-60}$ & $\frac{\text { Unabsor }}{\text { SXC }}$ & $\frac{\text { ed } F_{\mathrm{s}}, F_{\mathrm{h}}}{\mathrm{HXC}}$ & $\begin{array}{c}N_{\mathrm{H}} \\
\left(10^{22} \mathrm{~cm}^{-2}\right)\end{array}$ & $\begin{array}{c}k T \\
(\mathrm{keV})\end{array}$ & $\begin{array}{c}R \\
(\mathrm{~km})\end{array}$ & $\Gamma_{\mathrm{s}}$ & $\Gamma_{\mathrm{h}}$ & $\chi_{\nu}^{2}(\mathrm{dof})$ \\
\hline \multicolumn{12}{|c|}{ Suzaku observations } \\
\hline $1806-20$ & 401092010 & $12.6_{-0.1}^{+0.1}$ & $33.7(4.4)$ & $6.3_{-0.8}^{+0.9}$ & $58.4_{-1.4}^{+1.4}$ & $6.7(3)$ & $0.61(4)$ & 1.8 & $\ldots$ & $1.62(5)$ & $0.97(377)$ \\
\hline $1806-20$ & 401021010 & $10.5_{-0.2}^{+0.1}$ & $21.1(3.3)$ & $3.4_{-0.8}^{+0.9}$ & $50.1_{-2.6}^{+2.9}$ & $5.5(5)$ & $0.68(9)$ & 1.1 & $\ldots$ & $1.51(9)$ & $1.18(171)$ \\
\hline $1806-20$ & 402094010 & $8.9_{-0.2}^{+0.1}$ & $27.1(4.3)$ & $5.9_{-0.8}^{+1.0}$ & $42.2_{-1.7}^{+1.8}$ & $6.5(5)$ & $0.65(6)$ & 1.5 & $\ldots$ & $1.50(7)$ & $1.22(193)$ \\
\hline $1841-04$ & 401100010 & $19.4_{-0.1}^{+0.1}$ & $48.9(0.3)$ & $35.1_{-1.4}^{+1.4}$ & $50.9_{-2.1}^{+2.2}$ & $2.5(1)$ & $0.27(1)$ & 21.1 & $3.41(10)$ & $0.87(8)$ & $1.22(2087)$ \\
\hline $1900+14$ & 401022010 & $5.3_{-0.5}^{+0.5}$ & $20.6(5.5)$ & $4.6_{-0.6}^{+0.1}$ & $25.0_{-3.4}^{+3.2}$ & $1.8(3)$ & $0.57(2)$ & 2.5 & $\ldots$ & $0.96(14)$ & $1.13(44)$ \\
\hline $1900+14$ & 404077010 & $4.3_{-0.1}^{+0.0}$ & $16.5(3.5)$ & $4.5_{-0.3}^{+0.3}$ & $26.3_{-2.5}^{+2.9}$ & $1.9(1)$ & $0.52(2)$ & 3.0 & $\ldots$ & $0.78(9)$ & $1.34(57)$ \\
\hline $1714-38$ & 501007010 & $1.7_{-0.1}^{+0.1}$ & $\ldots$ & $5.0_{-0.3}^{+0.5}$ & $\ldots$ & $3.5(1)$ & $0.24(4)$ & 15.7 & $3.25(8)$ & $\ldots$ & $1.28(179)$ \\
\hline $1708-40$ & 404080010 & $38.1_{-0.2}^{+0.5}$ & $24.4(4.4)$ & $69.1_{-3.3}^{+3.5}$ & $28.4_{-2.7}^{+2.8}$ & $1.3(1)$ & $0.26(2)$ & 14.2 & $3.48(9)$ & $0.67(24)$ & $0.98(394)$ \\
\hline $1708-40$ & 405076010 & $35.8_{-0.1}^{+0.4}$ & $24.4(4.0)$ & $55.0_{-2.7}^{+4.0}$ & $33.4_{-2.2}^{+2.1}$ & $1.2(1)$ & $0.30(1)$ & 9.5 & $3.80(21)$ & $1.14(16)$ & $1.05(540)$ \\
\hline $1048-59$ & 403005010 & $9.9_{-0.1}^{+0.1}$ & $<13.2$ & $12.3_{-0.2}^{+0.2}$ & $<19.5$ & $0.47(3)$ & $0.45(1)$ & 4.7 & $4.88(20)$ & $\ldots$ & $1.32(78)$ \\
\hline $0142+61$ & 402013010 & $122.0_{-0.1}^{+0.1}$ & $35.1(6.4)$ & $185.1_{-0.7}^{+0.7}$ & $38.3_{-2.3}^{+2.3}$ & $0.61(1)$ & $0.28(1)$ & 19.0 & $4.68(2)$ & $0.24(7)$ & $1.43(1725)$ \\
\hline $0142+61$ & 404079010 & $115.6_{-0.2}^{+0.2}$ & $26.2(5.8)$ & $176.8_{-1.5}^{+1.5}$ & $27.1_{-2.2}^{+2.3}$ & $0.62(1)$ & $0.28(1)$ & 18.6 & $4.71(4)$ & $0.39(14)$ & 1.15 (1401) \\
\hline $0142+61$ & 406031010 & $108.3_{-0.9}^{+1.2}$ & $24.3(3.7)$ & $164.8_{-4.4}^{+4.3}$ & $41.3_{-4.0}^{+4.1}$ & $0.63(3)$ & $0.28(1)$ & 18.0 & $4.65(10)$ & $0.32(21)$ & $1.08(1062)$ \\
\hline $0142+61$ & 408011010 & $107.8_{-0.4}^{+0.4}$ & $19.1(2.8)$ & $162.7_{-1.4}^{+1.4}$ & $\begin{array}{l}32.9_{-2.2}^{+2.3} \\
\end{array}$ & $0.60(1)$ & $0.28(1)$ & 17.9 & $4.81(4)$ & $0.26(11)$ & $1.37(838)$ \\
\hline $2259+58$ & 404076010 & $30.4_{-0.1}^{+0.1}$ & $<10.1$ & $44.8_{-0.5}^{+0.5^{t}}$ & $<14.9$ & $0.55(1)$ & $0.29(1)$ & 7.8 & $4.85(4)$ & $\ldots$ & $1.20(510)$ \\
\hline $1818-15$ & 406074010 & $1.0_{-0.1}^{+0.1}$ & $\ldots$ & $1.4_{-0.1}^{+0.1}$ & $\ldots$ & $2.1(21)$ & $1.61(6)$ & 0.1 & $\ldots$ & $\ldots$ & $1.31(83)$ \\
\hline $1547-54$ & 903006010 & $59.7_{-0.8}^{+0.9}$ & $110.2(5.2)$ & $50.6_{-1.7}^{+1.7}$ & $158.7_{-3.3}^{+3.3}$ & $2.8(1)$ & $0.67(2)$ & 1.9 & $\ldots$ & $1.53(4)$ & $1.29(140)$ \\
\hline $1547-54$ & 405024010 & $11.1_{-0.2}^{+0.1}$ & $13.5(3.3)$ & $17.5_{-0.6}^{+0.6}$ & $23.4_{-2.4}^{+2.5}$ & $2.8(1)$ & $0.62(1)$ & 1.3 & $\ldots$ & $1.15(12)$ & $1.23(69)$ \\
\hline $0501+45$ & 903002010 & $36.2_{-0.2}^{+0.3}$ & $28.1(6.5)$ & $42.2_{-0.8}^{+0.6}$ & $30.4_{-3.7}^{+4.0}$ & $0.40(2)$ & $0.49(1)$ & 2.7 & $4.35(17)$ & $0.10(35)$ & $1.23(206)$ \\
\hline $0501+45$ & 404078010 & $2.9_{-0.1}^{+0.1}$ & $<20.9$ & $3.8_{-0.2}^{+0.3}$ & $<30.8$ & $0.44(10)$ & $0.30(3)$ & 2.2 & $4.16(27)$ & $\ldots$ & $0.92(77)$ \\
\hline $0501+45$ & 405075010 & $1.7_{-0}^{+0}$ & $<16.2$ & $2.0_{-0.2}^{+0.2}$ & $<23.9$ & $0.24(13)$ & $0.30(4)$ & 1.6 & $4.20(45)$ & $\ldots$ & $0.80(34)$ \\
\hline $0501+45$ & 408013010 & $1.7_{-0.1}^{+0.1}$ & $<12.7$ & $2.3_{-0.1}^{+0.1}$ & $<18.8$ & $0.41(7)$ & $0.26(2)$ & 2.3 & $3.79(15)$ & $\ldots$ & $0.96(367)$ \\
\hline $1833-08$ & 904006010 & $3.8_{-0.1}^{+0.1}$ & $17.6(4.5)$ & $7.9_{-0.2}^{+0 . \frac{1}{+}}$ & $35.6_{-8.8}^{+9.3}$ & $9.6(5)$ & $1.08(4)$ & 0.7 & $\ldots$ & $-0.38(40)$ & $1.37(167)$ \\
\hline $1647-45$ & 901002010 & $27.2_{-0.1}^{+0.1}$ & $\ldots$ & $45.9_{-0.5}^{+0.5}$ & $\ldots$ & $1.7(1)$ & $0.49(1)$ & 3.4 & $4.39(6)$ & $\ldots$ & $1.26(248)$ \\
\hline $1822-16$ & 906002010 & $18.2_{-0.3}^{+0.4}$ & $<4.9$ & $18.5_{-0.4}^{+0.5}$ & $<7.2$ & $0.02(4)$ & $0.54(2)$ & 0.7 & $5.86(71)$ & $\ldots$ & $1.41(59)$ \\
\hline \multicolumn{12}{|c|}{ NuSTAR observations } \\
\hline $1841-04$ & 30001025004 & $23.6_{-0.1}^{+0.1}$ & $35.2(0.6)$ & $29.9_{-0.1}^{+0.1}$ & $48.6_{-0.5}^{+0.5}$ & $2.3(1)$ & $0.28(3)$ & 18.1 & $3.45(6)$ & $1.13(2)$ & $1.15(1493)$ \\
\hline $0142+61$ & 30001023002 & $114.6_{-0.1}^{+0.3}$ & $21.7(0.3)$ & $167.1_{-0.1}^{+0.1}$ & $27.4_{-0.4}^{+0.4}$ & $0.58(2)$ & $0.30(1)$ & 15.8 & $4.84(2)$ & $0.61(2)$ & $1.11(765)$ \\
\hline $2259+58$ & 30001026002 & $28.9_{-0.9}^{+0.1}$ & $3.1(0.3)$ & $51.1_{-2.3}^{+2.8}$ & $3.7_{-0.3}^{+0.3}$ & $0.84(4)$ & $0.28(1)$ & 8.9 & $4.95(6)$ & $0.58(13)$ & $1.07(404)$ \\
\hline $2259+58$ & 30001026007 & $24.9_{-2.3}^{+0.1}$ & $3.0(0.3)$ & $42.0_{-3.7}^{+4.3}$ & $3.4_{-0.2}^{+0.2}$ & $0.78(7)$ & $0.30(1)$ & 7.0 & $5.06(8)$ & $0.66(14)$ & $0.94(222)$ \\
\hline $1048-59$ & 2013-07 & $\sim 8 . \overline{9}$ & $\lesssim 6.7$ & $\sim 18.3$ & $\mathrm{~N} / \mathrm{A}$ & \multicolumn{6}{|c|}{ from Yang et al. (2015) } \\
\hline
\end{tabular}

a $F_{1-10}$ and $F_{15-60}:$ Absorbed $1-10 \mathrm{keV}$ and $15-60 \mathrm{keV}$ fluxes $\left(10^{-12} \mathrm{erg} \mathrm{s}^{-1} \mathrm{~cm}^{-2}\right)$

b Unabsorbed flx $F_{\mathrm{s}}$ and $F_{\mathrm{h}}$ of the SXC and HXC in the 1-60 keV band $\left(10^{-12} \mathrm{erg} \mathrm{s}^{-1} \mathrm{~cm}^{-2}\right)$.

${ }^{\mathrm{c}}$ Radius is evaluated from $R=0.09643 \times(d / \mathrm{kpc})(T / \mathrm{keV})^{-2}\left(F_{\mathrm{s}} / 10^{-11} \mathrm{erg} \mathrm{s}^{-1} \mathrm{~cm}^{-1}\right)^{0.5}$

Kaspi \& Boydstun 2010).

Based on our updated sample shown in Figure8, we revised these correlations. Figure 10 top-left panel shows the ratio of absorbed fluxes, $\eta=F_{15-60} / F_{1-10}$, as a function of $\dot{P}$, which is derived from the pulsar timing information independently from the spectroscopy. The Spearman's rank-order test of this correlation gives a significantly high value, $r_{s}=0.94$. The correlation is fitted as,

$$
\begin{aligned}
\eta & =F_{15-60} / F_{1-10} \\
& =(0.59 \pm 0.07) \times\left(\dot{P} / 10^{-11} \mathrm{~s} \mathrm{~s}^{-1}\right)^{0.51 \pm 0.05},
\end{aligned}
$$

using the Bayesian method in Kelly (2007) (linmix package) to account for measurement errors and intrinsic scatter of the data. While the correlations is derived from the spectral analyses below $70 \mathrm{keV}$, the potential HXC cutoff does not strongly affect the correlation (Paper I). Due to limited photon statistics of several Suzaku sources, we can only study total emission. Deconvolution into pulsed and un-pulsed components (Kuiper et al. 2012) will be reported in future publications.
As shown in $\nu F_{\nu}$ plots (Figure 8), the HXC largely contributed to the SXC band below $\lesssim 10 \mathrm{keV}$ in stronger $B_{\mathrm{d}}$ objects (e.g., SGR 1806-20, SGR $1900+14$, and $1 \mathrm{E}$ 1547.0-5408). To remove this mixing, and to eliminate the effects of the photo-absorption, we also define the absorption-corrected luminosity ratio between the two components, $\xi=L_{\mathrm{h}} / L_{\mathrm{s}}$ (listed in Table 6), in the same way as Paper I. The Spearman's rank-order significance becomes $r_{s}=0.97$. The correlation slope of $\xi$ becomes steeper than those of $\eta$ as,

$$
\begin{aligned}
\xi & =L_{\mathrm{h}} / L_{\mathrm{s}} \\
& =(0.62 \pm 0.07) \times\left(\dot{P} / 10^{-11} \mathrm{~s} \mathrm{~s}^{-1}\right)^{0.72 \pm 0.05} .
\end{aligned}
$$

This is shown in the top-right panel of Figure 10. On these correlations, we revised timing information $(P$ and $\dot{P}$ ) from Paper I, referring to the McGill cata$\log$ (Olausen \& Kaspi 2013), and further added $N u S$ TAR observations. Data points of canonical AXPs $4 \mathrm{U} \quad 0142+61$ and $1 \mathrm{E}$ 1841-045 are consistent with those with Suzaku, and the new HXC detection from 1E 2259+586 (Vogel et al. 2014) falls on the correlation. 
Table 6

X-ray luminosities of the soft and hard components from magnetars measured with Suzaku.

\begin{tabular}{|c|c|c|c|c|c|c|c|c|c|c|c|c|}
\hline Name & ObsID & $\begin{array}{c}\text { Month } \\
\text { yyyy-mm }\end{array}$ & $\begin{array}{l}P \\
(\mathrm{~s}) \\
\end{array}$ & $\dot{P}_{11}$ & $B_{14}$ & $\begin{array}{c}\tau \\
(\mathrm{kyr}) \\
\end{array}$ & $L_{\mathrm{sd}}$ & $L_{\mathrm{S}}$ & $L_{\mathrm{h}}$ & $\begin{array}{c}L_{\mathrm{x}} \\
\text { (total) } \\
\end{array}$ & $\begin{array}{l}\text { Abs. HR } \\
\left(\frac{F_{15-60}}{F_{1-10}}\right) \\
\end{array}$ & $\begin{array}{c}\mathrm{HR} \\
\left(\xi=L_{\mathrm{h}} / L_{\mathrm{s}}\right)\end{array}$ \\
\hline \multicolumn{13}{|c|}{ Suzaku } \\
\hline $1806-20$ & 401092010 & 2006-09 & 7.548 & 49.5 & 19.6 & 0.2 & 4.6 & $5.7_{-0.1}^{+0.8}$ & $53.0_{-0.1}^{+1.2}$ & $58.8_{1.5}^{+1.5}$ & $2.7_{-0.4}^{+0.4}$ & $9.3_{-1.2}^{+1.4}$ \\
\hline $1806-20$ & 401021010 & 2007-03 & $\ldots$ & $\ldots$ & $\ldots$ & $\ldots$ & $\ldots$ & $3.1_{-0.1}^{+0.9}$ & $45.5_{-0.1}^{+2.6}$ & $48.6_{2.5}^{+2.8}$ & $2.0_{-0.3}^{+0.3}$ & $14.7_{-3.7}^{+4.2}$ \\
\hline $1806-20$ & 402094010 & $2007-10$ & $\ldots$ & $\ldots$ & $\ldots$ & $\ldots$ & $\ldots$ & $5.3_{-0.1}^{+0.9}$ & $38.4_{-0.1}^{+1.1}$ & $43.7_{1.7}^{+1.5}$ & $3.0_{-0.5}^{+0.3}$ & $7.2_{-1.3}^{+1.7}$ \\
\hline $1841-04$ & 401100010 & 2006-04 & 11.789 & 4.1 & 7.0 & 4.6 & 0.100 & $30.5_{-0.1}^{+1.2}$ & $44.1_{-0.1}^{+1.9}$ & $74.6_{2.2}^{+2.2}$ & $2.5_{-0.1}^{+0.1}$ & $1.4_{-0.1}^{+0.1}$ \\
\hline $1900+14$ & 401022010 & 2006-04 & 5.200 & 9.2 & 7.0 & 0.9 & 2.6 & $8.7_{-0.1}^{+0.2}$ & $46.9_{-0.1}^{+6.0}$ & $55.5_{6.5}^{+6.0}$ & $3.9_{-1.1}^{+1.1}$ & $5.4_{-1.0}^{+0.7}$ \\
\hline $1900+14$ & 404077010 & 2009-04 & $\ldots$ & $\ldots$ & $\ldots$ & $\ldots$ & $\ldots$ & $8.4_{-0.1}^{+0.5}$ & $49.3_{-0.1}^{+5.5}$ & $57.7_{4.8}^{+5.5}$ & $3.8_{-0.8}^{+0.1}$ & $5.9_{-0.7}^{+0.7}$ \\
\hline $1714-38$ & 501007010 & 2006-08 & 3.825 & 6.4 & 5.0 & 0.9 & 4.6 & $10.5_{-0.1}^{+0.9}$ & & $\left(=L_{\mathrm{s}}\right)$ & & \\
\hline $1708-40$ & 404080010 & 2009-08 & 11.005 & 1.9 & 4.7 & 9.0 & 0.058 & $12.0_{-0.1}^{+0.6}$ & $4.9_{-0.1}^{+0.5}$ & $16.9_{0.7}^{+0.8}$ & $0.64_{-0.12}^{+0.12}$ & $0.41_{-0.04}^{+0.05}$ \\
\hline $1708-40$ & 405076010 & 2010-09 & $\ldots$ & $\ldots$ & $\ldots$ & $\ldots$ & $\ldots$ & $9.5_{-0.1}^{+0.1}$ & $5.8_{-0.1}^{+0.4}$ & $15.3_{0.6}^{+0.8}$ & $0.68_{-0.11}^{+0.11}$ & $0.61_{-0.05}^{+0.06}$ \\
\hline $1048-59$ & 403005010 & 2008-11 & 6.458 & 2.3 & 3.9 & 4.6 & 0.33 & $11.9_{-0.1}^{+0.2}$ & $<19.0$ & $\left(=L_{\mathrm{S}}\right)$ & $<1.3$ & $\begin{array}{l}<2.0 \\
<0\end{array}$ \\
\hline $0142+61$ & 402013010 & 2007-08 & 8.689 & 0.20 & 1.3 & 68.1 & 0.012 & $28.8_{-0.1}^{+0.1}$ & $6.0_{-0.1}^{+0.4}$ & $34.7_{0.4}^{+0.4}$ & $0.29_{-0.05}^{+0.05}$ & $0.21_{-0.01}^{+0.01}$ \\
\hline $0142+61$ & 404079010 & 2009-08 & $\ldots$ & $\ldots$ & $\ldots$ & $\ldots$ & $\ldots$ & $27.5_{-0.1}^{+0 . \frac{1}{2}}$ & $4.2_{-0.1}^{+0.1}$ & $31.7_{0.4}^{+0.4}$ & $0.23_{-0.05}^{+0.05}$ & $0.15_{-0.01}^{+0.01}$ \\
\hline $0142+61$ & 406031010 & 2011-09 & $\ldots$ & $\ldots$ & $\ldots$ & $\ldots$ & $\ldots$ & $25.6_{-0.1}^{+0.7}$ & $6.4_{-0.1}^{+0.6}$ & $32.1_{0.9}^{+1.0}$ & $0.22_{-0.03}^{+0.03}$ & $0.25_{-0.03}^{+0.03}$ \\
\hline $0142+61$ & 408011010 & 2013-07 & $\ldots$ & $\ldots$ & $\ldots$ & $\ldots$ & $\ldots$ & $25.3_{-0.1}^{+0 . \frac{1}{2}}$ & $5.1_{-0.1}^{+0.1}$ & $30.4_{0.4}^{+0.4}$ & $0.18_{-0.03}^{+0.03}$ & $0.20_{-0.01}^{+0.01}$ \\
\hline $2259+58$ & 404076010 & 2009-05 & 6.979 & 0.048 & 0.59 & 229.0 & 0.0057 & $5.5_{-0.1}^{+0.1}$ & $<1.8$ & $\left(=L_{\mathrm{S}}\right)$ & $<0.3$ & $<0.5$ \\
\hline $1818-15$ & 406074010 & 2011-10 & 2.482 & 0.80 & 1.4 & 4.9 & 2.1 & $0.30_{-0.01}^{+0.02}$ & $\ldots$ & $\left(=L_{\mathrm{S}}\right)$ & $\ldots$ & $\ldots$ \\
\hline $1547-54$ & 903006010 & 2009-01 & 2.072 & 4.8 & 3.2 & 0.7 & 21.4 & $9.3_{-0.1}^{+0.3}$ & $29.1_{-0.1}^{+0.6}$ & $38.4_{0.7}^{+0.7}$ & $1.8_{-0.1}^{+0.1}$ & $3.1_{-0.1}^{+0.1}$ \\
\hline $1547-54$ & 405024010 & 2010-08 & $\ldots$ & $\ldots$ & $\ldots$ & $\ldots$ & $\ldots$ & $3.2_{-0.1}^{+0.1}$ & $4.3_{-0.1}^{+0.15}$ & $7.5_{0.4}^{+0.5}$ & $1.2_{-0.3}^{+0.1}$ & $1.3_{-0.1}^{+0.2}$ \\
\hline $0501+45$ & 903002010 & 2008-08 & 5.762 & 0.59 & 1.9 & 15.4 & 0.12 & $5.5_{-0.1}^{+0.1}$ & $4.0_{-0.1}^{+0.5}$ & $9.5_{0.5}^{+0.5}$ & $0.78_{-0.18}^{+0.18}$ & $0.72_{-0.09}^{+0.10}$ \\
\hline $0501+45$ & 404078010 & 2009-08 & $\ldots$ & $\ldots$ & $\ldots$ & $\ldots$ & $\ldots$ & $0.50_{-0.01}^{+0.04}$ & $<4.0$ & $\left(=L_{\mathrm{S}}\right)$ & $<7.3$ & $<10.7$ \\
\hline $0501+45$ & 405075010 & 2010-09 & $\ldots$ & $\ldots$ & $\ldots$ & $\ldots$ & $\ldots$ & $0.26_{-0.01}^{+0.03}$ & $<3.1$ & $\left(=L_{\mathrm{S}}\right)$ & $<9.6$ & $<14.2$ \\
\hline $0501+45$ & 408013010 & 2013-08 & $\ldots$ & $\ldots$ & $\ldots$ & $\ldots$ & $\ldots$ & $0.30_{-0.01}^{+0.02}$ & $<2.5$ & $\left(=L_{\mathrm{S}}\right)$ & $<7.4$ & $<10.9$ \\
\hline $1833-08$ & 904006010 & 2010-03 & 7.565 & 0.35 & 1.6 & 34.3 & 0.032 & $7.6_{-0.1}^{+0.31}$ & $34.6_{-0.1}^{+9.0}$ & $42.3_{8.6}^{+9.0}$ & $4.7_{-1.2}^{+1.2}$ & $4.5_{-1.1}^{+1.2}$ \\
\hline $1647-45$ & 901002010 & 2006-09 & 10.61 & $\cdots$ & $\cdots$ & $\cdots$ & $\cdots$ & $8.4_{-0.1}^{+0.1}$ & $\cdots$ & $\left(=L_{\mathrm{S}}\right)$ & .. & $\cdots$ \\
\hline $1822-16$ & 906002010 & 2011-09 & 8.438 & 0.0021 & 0.14 & 6250.0 & 0.00014 & $0.57_{-0.01}^{+0.01}$ & $<0.2$ & $\left(=L_{\mathrm{S}}\right)$ & $<0.3$ & $<0.4$ \\
\hline \multicolumn{13}{|c|}{ NuSTAR } \\
\hline $1841-04$ & 30001025004 & 2013-09 & 11.789 & 4.1 & 7.0 & 4.6 & 0.100 & $25.9_{-0.1}^{+0.1}$ & $42.1_{-0.1}^{+0.4}$ & $68.1_{0.4}^{+0.4}$ & $1.5_{-0.1}^{+0.1}$ & $1.6_{-0.1}^{+0.1}$ \\
\hline $0142+61$ & 30001023002 & 2014-01 & 8.689 & 0.20 & 1.3 & 68.1 & 0.012 & $26.0_{-0.1}^{+0.1}$ & $4.3_{-0.1}^{+0.1}$ & $30.2_{0.1}^{+0.1}$ & $0.19_{-0.01}^{+0.91}$ & $0.16_{-0.01}^{+0.01}$ \\
\hline $2259+58$ & 30001026002 & 2013-04 & 6.979 & 0.048 & 0.59 & 229.0 & 0.0057 & $6.3_{-0.1}^{+0.3}$ & $0.5_{-0.1}^{+0.1}$ & $6.7_{0.3}^{+0.3}$ & $0.11_{-0.01}^{+0.01}$ & $0.07_{-0.01}^{+0.01}$ \\
\hline $2259+58$ & 30001026007 & 2013-05 & $\ldots$ & $\ldots$ & $\ldots$ & $\ldots$ & $\ldots$ & $5.2_{-0.1}^{+0 . \frac{1}{6}}$ & $0.4_{-0.1}^{+0.1}$ & $5.6_{0.5}^{+0.6}$ & $0.12_{-0.02}^{+0.01}$ & $0.08_{-0.01}^{+0.01}$ \\
\hline $1048-59$ & 30001024002 & 2013-07 & 6.458 & 2.3 & 3.9 & 4.6 & 0.33 & $17.8_{-0.1}^{+0.1}$ & $<6.1_{-0.1}^{+0.1}$ & $23.9_{0.0}^{+0.0}$ & $<0.74$ & $<0.34$ \\
\hline
\end{tabular}

Note. - Pulsar timing information: spin period $P(\mathrm{~s})$, period derivative $\dot{P}_{11}=\dot{P} /\left(10^{-11} \mathrm{~s} \mathrm{~s}^{-1}\right)$, surface magnetic field $B 14=B /\left(10^{14} \mathrm{G}\right)$, and spin-down luminosity $L_{\mathrm{sd}}=4 \pi I^{2} \dot{P} / P^{3}=3.2 \times 10^{33} \dot{P}_{11}(P / 5 \mathrm{~s})^{-3} \mathrm{erg} \mathrm{s}^{-1}$, where $I=10^{45} \mathrm{~g} \mathrm{~cm}^{2}$ is the neutron star momentum of inertia. Spectral information: The 1-60 keV luminosity of the SXC and HXC, $L_{\mathrm{s}}, L_{\mathrm{h}}$, and their toral $L_{\mathrm{x}}=L_{\mathrm{s}}+L_{\mathrm{h}}$. All the luminosities, $L_{\mathrm{sd}}, L_{\mathrm{s}}, L_{\mathrm{h}}$, and $L_{\mathrm{x}}$ are shown in an unit of $10^{34} \mathrm{erg} \mathrm{s}^{-1}$.

Hardness ratio (HR): Evaluated from absorbed fluxes of $15-60 \mathrm{keV}$ and $1-10 \mathrm{keV}\left(\eta=F_{15-60} / F_{1-10}\right)$ or luminosities $\left(\xi=L_{\mathrm{h}} / L_{\mathrm{S}}\right)$ after correcting absorption.

The Galactic center source SGR J1745-29, though not shown in Figure 10, is also expected to follow the relation since its wide-band spectrum resembles that of 1E 1547.0-5408 (Mori et al. 2013).

The above correlation to the directly measured quantity $\dot{P}$ can be converted to correlations to $B_{\mathrm{d}}$ and $\tau_{\mathrm{d}}$, although the additional two relations are not independent to Eq. (2) since $B_{\mathrm{d}}$ and $\tau_{\mathrm{c}}$ are estimated using combinations of the same $P$ and $\dot{P}$; i.e., $B_{\mathrm{d}} \propto P^{1 / 2} \dot{P}^{1 / 2}$ and $\tau_{\mathrm{c}} \propto P \dot{P}^{-1}$. Furthermore, considering the clustering of rotational periods of magnetars in a narrow range $(P=2-11 \mathrm{~s})$, Correlation of Eq. (2), $\xi \propto \dot{P}^{-k}(\mathrm{k} \sim 0.72)$, gives $\xi \propto B_{\mathrm{d}}^{2 k} \sim B_{\mathrm{d}}^{1.4}$ and $\xi \propto \tau_{\mathrm{c}}^{-k} \sim \tau_{\mathrm{c}}^{-0.72}$. These relations are shown in the middle and bottom panels in Figure 10, and same fitting procedures give,

$$
\begin{aligned}
& =(0.097 \pm 0.036) \times\left(B_{\mathrm{d}} / B_{\mathrm{QED}}\right)^{1.00 \pm 0.14} \\
& =(1.91 \pm 0.33) \times\left(\tau_{\mathrm{c}} / 1 \mathrm{kyr}\right)^{-0.48 \pm 0.05}
\end{aligned}
$$

and

$$
\begin{aligned}
\xi & =L_{\mathrm{h}} / L_{\mathrm{s}} \\
& =(0.050 \pm 0.022) \times\left(B_{\mathrm{d}} / B_{\mathrm{QED}}\right)^{1.41 \pm 0.16} \\
& =(3.25 \pm 0.50) \times\left(\tau_{\mathrm{c}} / 1 \mathrm{kyr}\right)^{-0.68 \pm 0.05} .
\end{aligned}
$$

The slopes of Eq. (5) and (6) are consistent with those from Paper I within error bars. The Suzaku upper limit for the second lowest $B_{\mathrm{d}}$-field source Swift J1822.3-1606 $\left(B_{\mathrm{d}}=1.4 \times 10^{13} \mathrm{G}\right.$, i.e., $\left.B_{\mathrm{d}} / B_{\mathrm{QED}} \sim 0.32\right)$ is also consistent with this picture. Thus we reconfirm, and reinforce, the evolution in $\eta$ and $\xi$ as reported in Paper I.

$$
\eta=F_{15-60} / F_{1-10}
$$




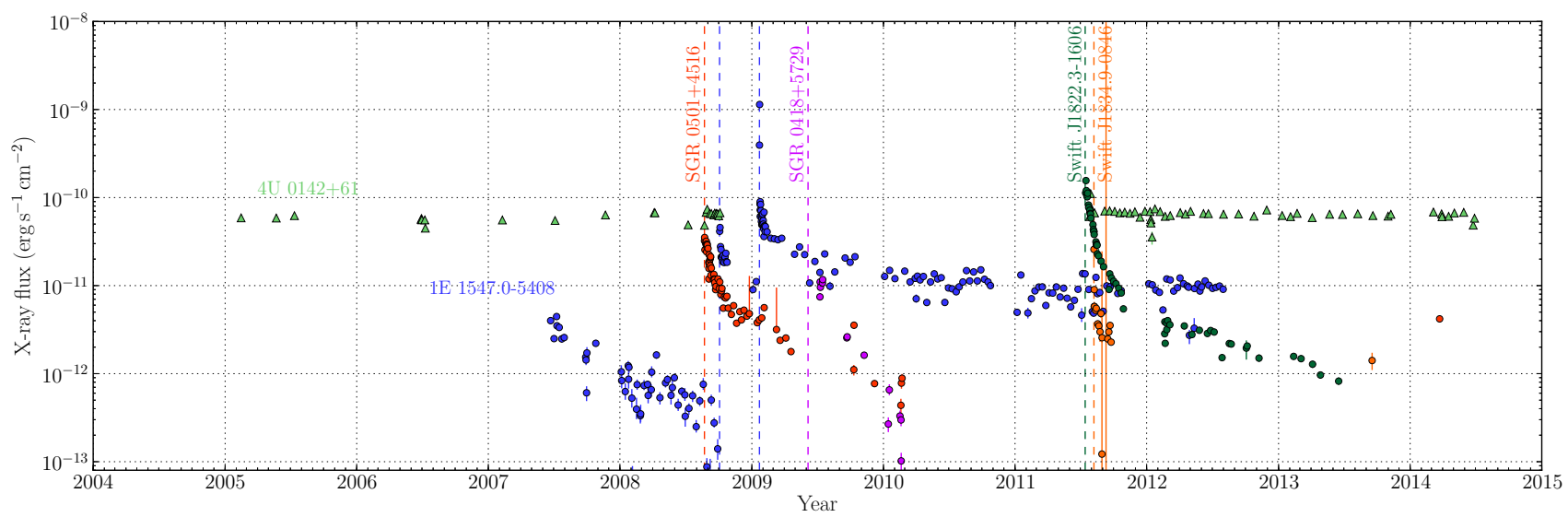

Figure 3. Examples of long-term histories in soft X-rays, during the Suzaku operation period, of persistently bright source (filled triangles) and transient objects (filled circles) monitored with Swift/XRT and RXTE/PCA. First short bursts (usually the discovery of transient sources) are indicated by the vertical dashed line for each outburst. The data process is described in $\S 3.2$.
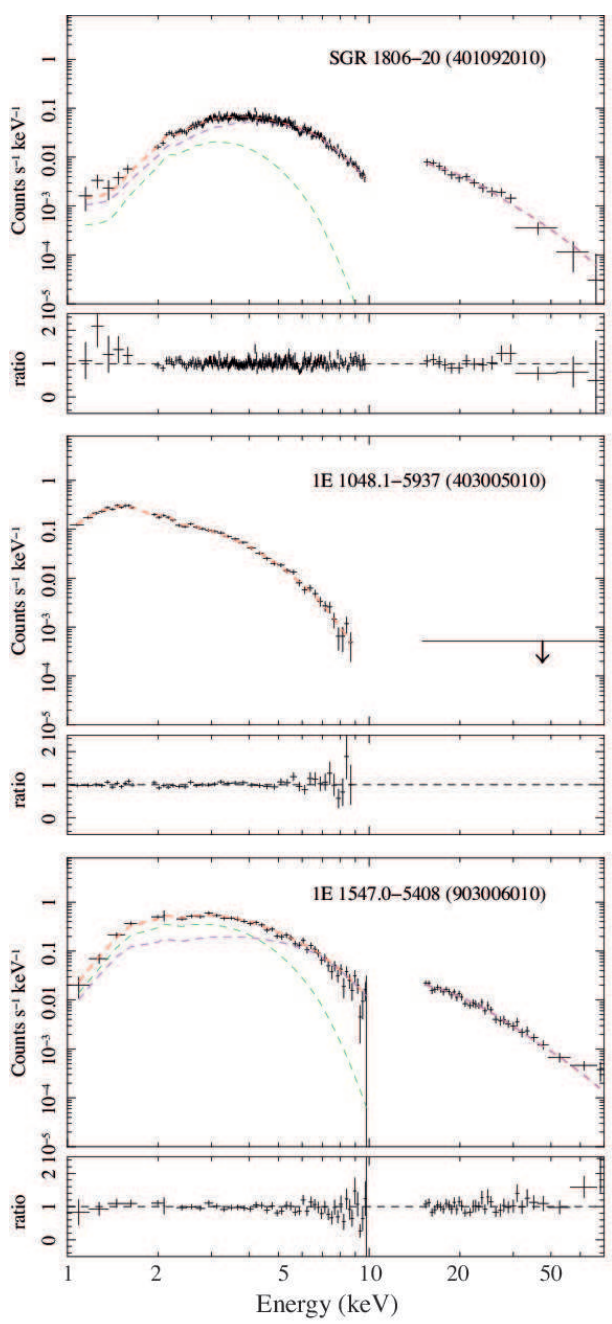
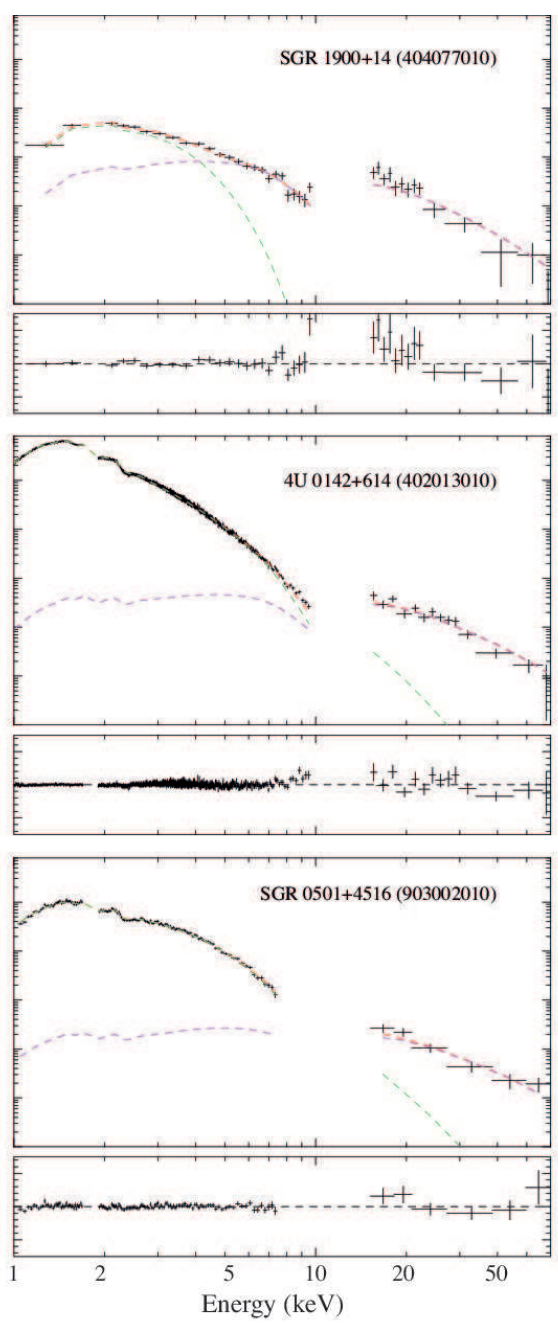
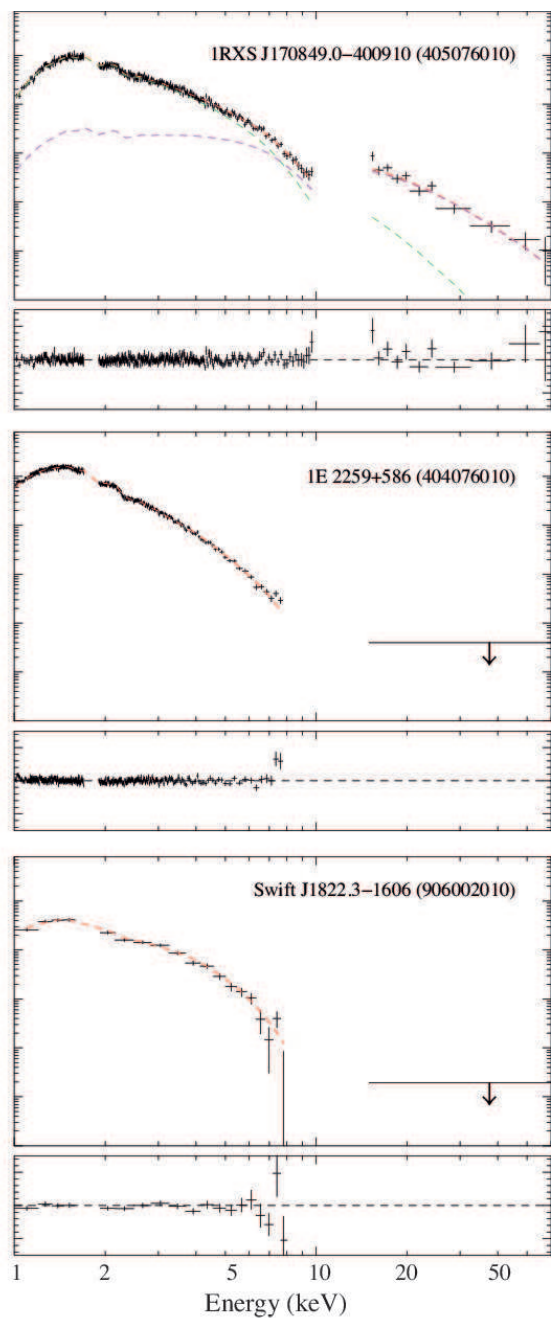

Figure 4. Examples of the XIS0+3 and HXD-PIN spectra of nine observations, peresented after subtracting the background, but not removing the instrumental responses. When the HXC is not detected, its $3 \sigma$ upper limits are shown. The XIS and HXD-PIN spectra are simultaneously fitted by the CBB model (green) plus the additional power-law for the HXC if detected. 


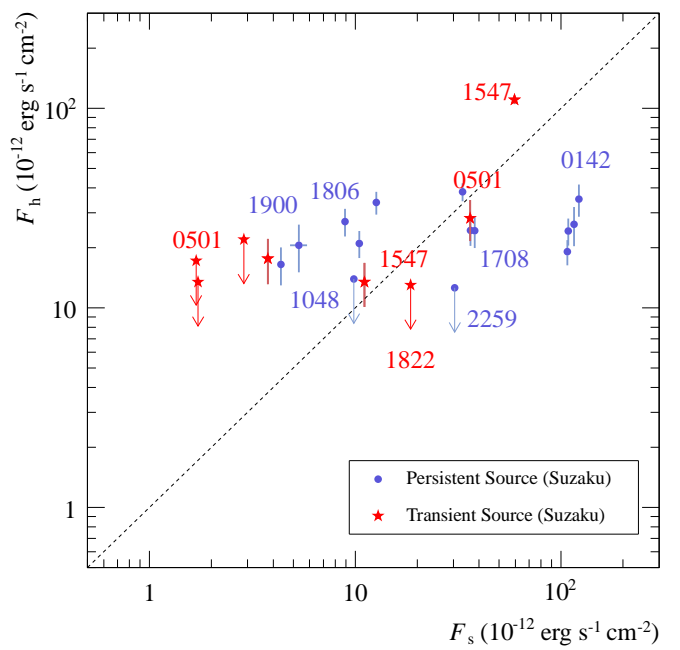

Figure 5. Comparison of the $1-10 \mathrm{keV}$ (XIS) and 15-60 keV (HXD-PIN) absorbed soft and hard fluxes, $F_{\mathrm{s}}$ and $F_{\mathrm{h}}$, respectively. The first four digits of the source names are indicated.

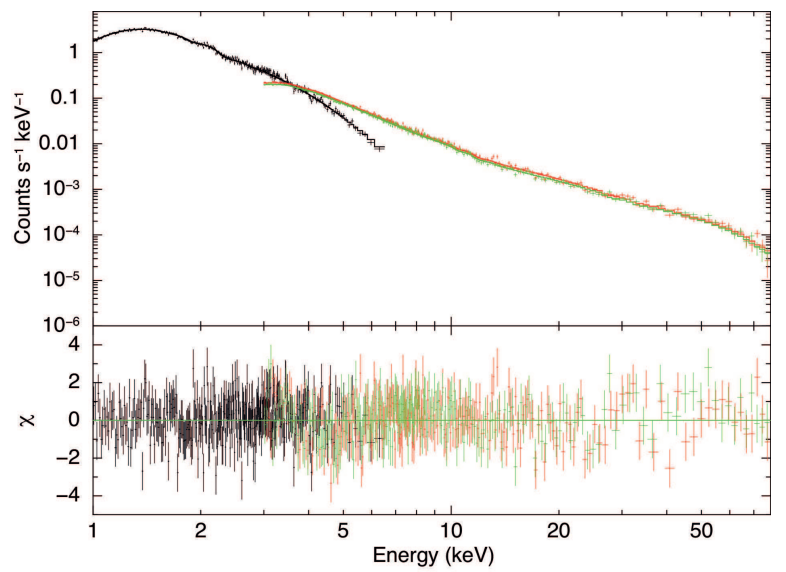

Figure 6. Background-subtracted NuSTAR $(>2.5 \mathrm{keV})$ and Swift $\mathrm{X}$-ray spectra of $4 \mathrm{U} 0142+61$ in 2014 March.

The second prediction of Paper I is the HXC spectral hardening toward weaker- $B_{\mathrm{d}}$ objects, as seen in representative $\nu F_{\nu}$ spectra in Figure 11 (top). Figure11 (bottom) also shows the HXC photon index $\Gamma_{\mathrm{h}}$ as a function of $B_{\mathrm{d}}$, which is fitted as

$$
\Gamma_{\mathrm{h}}=(0.40 \pm 0.11) \times\left(B_{\mathrm{d}} / B_{\mathrm{QED}}\right)^{0.35 \pm 0.09} .
$$

The $\Gamma_{h}$ values are stable on a long-time scale for persistently bright sources such as 4U 0142+61, SGR 1806-20, and $1 \mathrm{E}$ 1841-045, while some transients show slope change during the outbursts. This was also reported during the $\sim 400$ days INTEGRAL monitoring of $1 \mathrm{E} 1547.0-5408$ changing from $\Gamma \sim 1.4$ to $\sim 0.9$ (Kuiper et al. 2012). This figure clearly indicates a peculiar trend that relatively weaker HXC intensity sources show harder spectral slope of the HXC.

\subsubsection{Surface temperature $k T$ of $S X C$ vs. $B_{\mathrm{d}}$}

The surface temperature $T_{\mathrm{S}}$ of the SXC is plotted as a function of $B_{\mathrm{d}}$ in Figure 12 where we also added $T_{\mathrm{s}}$ of other isolated neutron stars from previous studies. This plot indicates 1) higher $T_{\mathrm{s}}$ of magnetars than that of

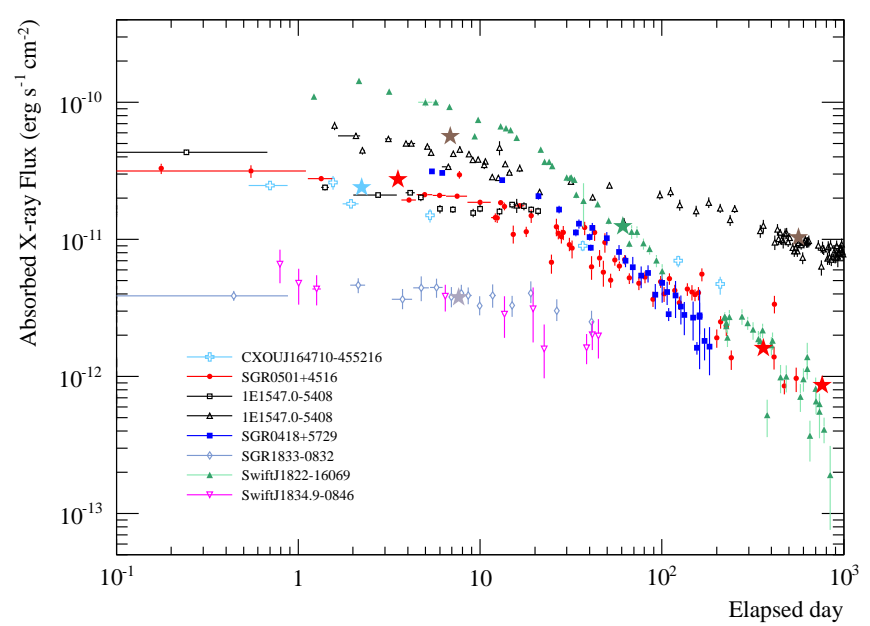

Figure 7. Absorbed $2-10 \mathrm{keV}$ flux decay of known 8 outbursts of transient SGRs and AXPs with time onsets defined at the first short burst detected by Swift/BAT or Fermi/GBM listed in Table 4 Star symbols (same color as the legend) represent Suzaku/XIS observations. The $2-10 \mathrm{keV}$ flux of $2 \times$ $10^{-11} \mathrm{erg} \mathrm{cm}^{-2} \mathrm{~s}^{-1}$ corresponds to $1 \mathrm{mCrab}$ intensity.

other isolated neutron stars, 2) a tendency of the positive correlation between $T_{\mathrm{S}}$ and $B_{\mathrm{d}}$ in the quiescent neutron star sample, and 3) increase of $T_{\mathrm{s}}$ during transient magnetar outbursts. All these properties suggest that the values of $T_{\mathrm{s}}$ reflect the effects of magnetic energy dissipation, which is an implicit but direct consequence of the magnetar hypothesis.

The magnetic field decay in the high field regime $B_{\mathrm{d}} \gtrsim 10^{13} \mathrm{G}$ (Goldreich \& Reisenegger 1992), postulated in the magnetar hypothesis, may be formulated as (Colpi et al. 2000; Dall'Osso et al. 2012)

$$
\frac{d B}{d t}=-a B^{1+\alpha},
$$

where $a$ and $\alpha$ are the normalization and decay index, respectively. The solution is obtained as

$$
B(t)=\frac{B_{0}}{\left(1+\alpha t / \tau_{B}\right)^{1 / \alpha}}
$$

with $\tau_{B}=\left(a B_{0}^{\alpha}\right)^{-1}$. This formula successfully explains the clustering of rotational period of SGRs and AXPs around 2-12s (Colpi et al. 2000), and resolves the overestimation of $\tau_{\mathrm{ch}}$ in $1 \mathrm{E} 2259+586$ when compared with the age derived from plasma diagnostics of the surrounding SNR CTB 109 Nakano et al. 2015, but see also Suwa \& Enoto 2014). Then, let us here assume that the surface temperature $T_{\mathrm{S}}$ is determined by a balance between the radiative cooling and heating by the magnetic energy dissipation of $d\left(B^{2} / 8 \pi\right) / d t$ in the crust. Following Pons et al. (2007), this is described as

$$
S \sigma T^{4}=-S \triangle R \frac{d}{d t}\left(\frac{B^{2}}{8 \pi}\right),
$$

where $S, \triangle R$, and $\sigma$ are the surface area, crust thickness, and the Stefan-Boltzmann constant, respectively. Combining this with Eq. (8), we derive

$$
4 \pi \sigma T^{4}=a \triangle R B^{2+\alpha},
$$



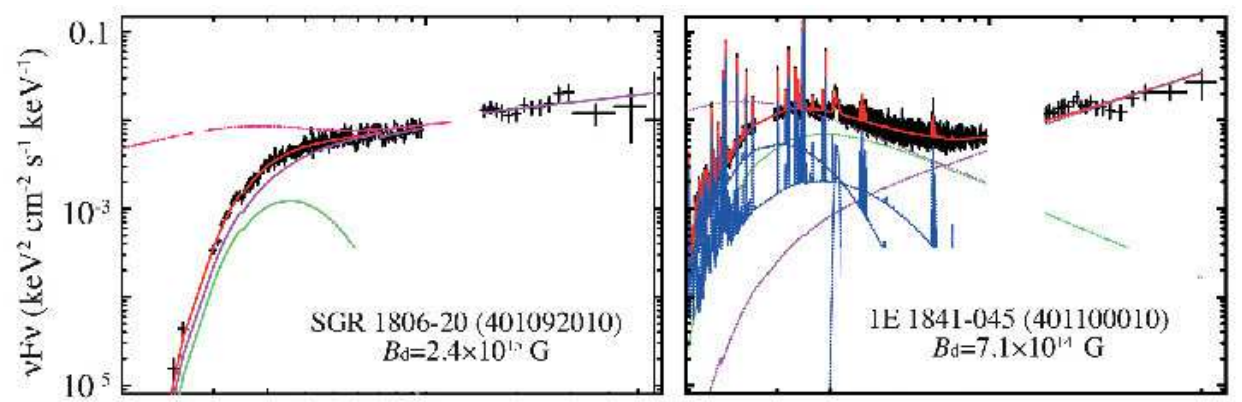

SGR and AXP spectra observed with Suzaku

- Soft X-ray Comp. Hard X-ray Comp.

in order of the decreasing dipole magnetic field $B$ d
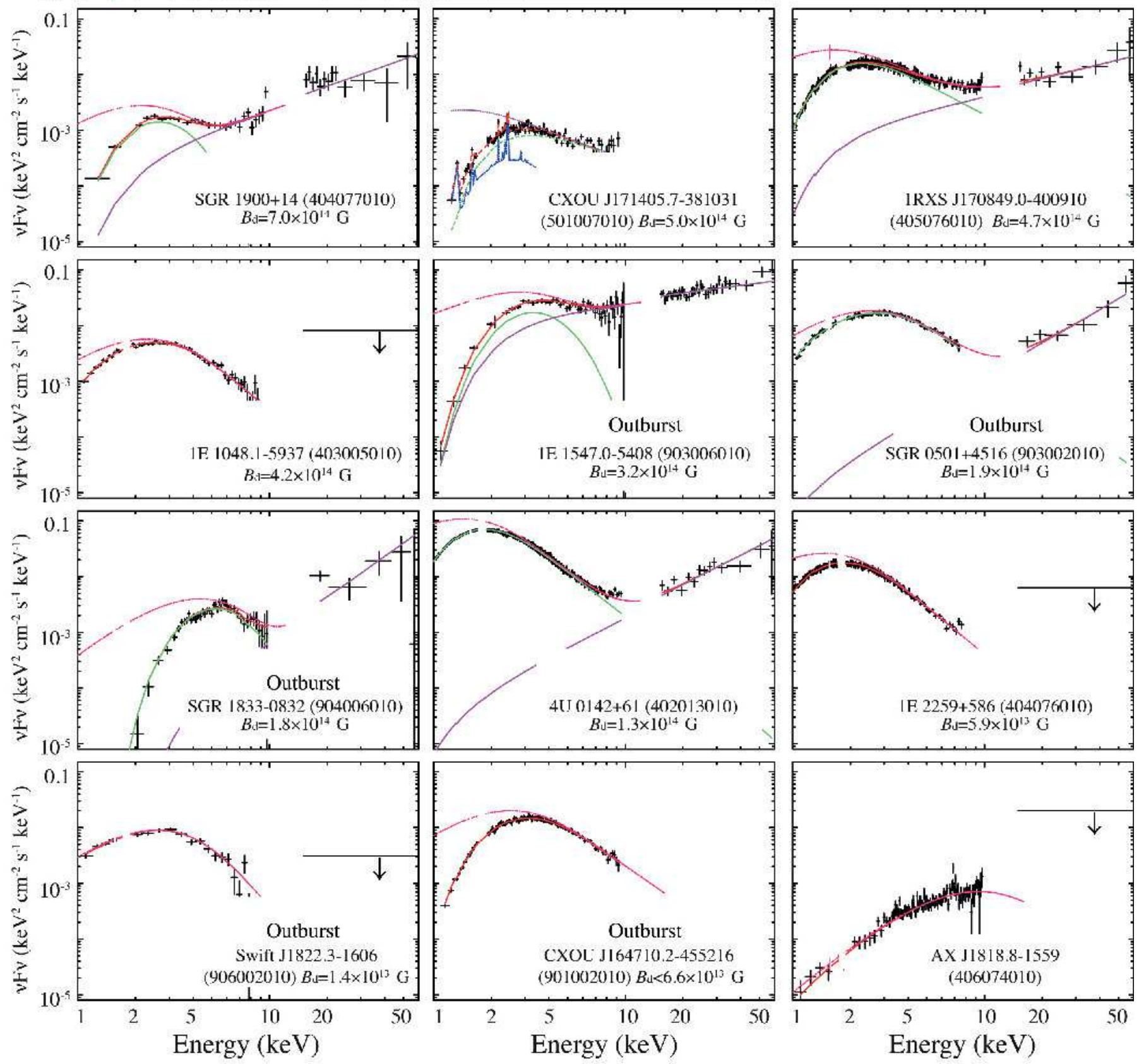

Figure 8. Suzaku $\nu F \nu$ spectra (black points) of persistent X-ray emission from fourteen SGRs and AXPs, deconvolved using the quasithermal CBB (green) and the hard PL (purple) model. The photoelectric absorption is included. Corresponding best-fit parameters are listed in Table 5 Absorption-corrected best-fit model is shown in pink. The spectra are shown in the order of decreasing dipole field $B_{\mathrm{d}}$ except for CXOU J164710.2-455216 (only the upper limit) and a magnetar candidate AX J1818.8-1559. The SNR components are shown in blue for 1E 1841-045 (Kes 73) and CXOU J171405.7-381031 (CTB 37B). 

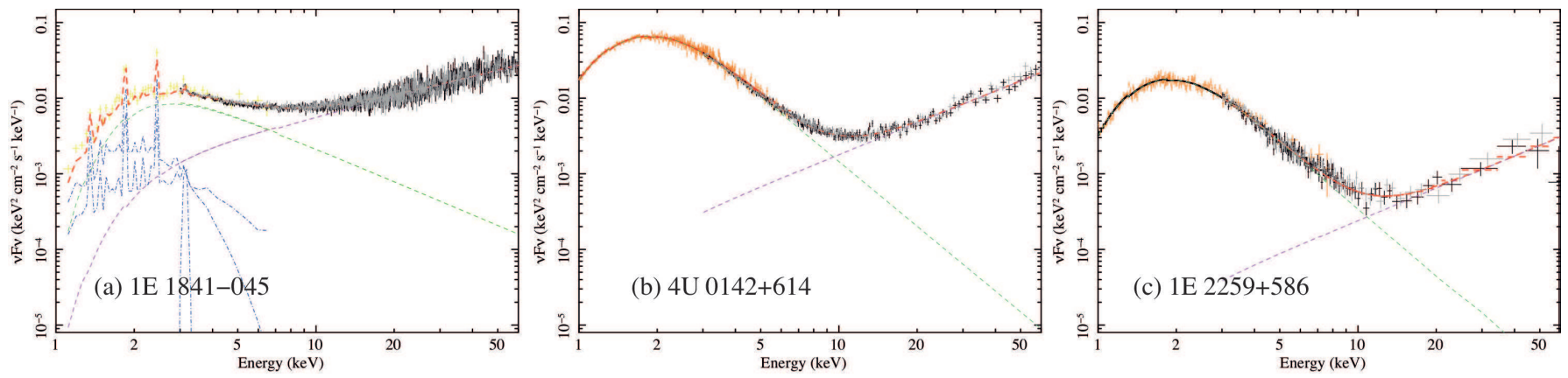

Figure 9. Same as Figure 8 but for NuSTAR observations of (a) 1E 1841-045 in 2013 September, (b) 4 U $0142+61$ in 2014 March, and (c) $1 \mathrm{E} 2259+586$.

or the relation of $T \propto B^{1 / 2+\alpha / 4}$. The slope of Figure 12 is close to a range, $\alpha \sim 1-2$, estimated from comparisons between pulsar and SNR ages (Nakano et al. 2015). This is another support for the magnetar hypothesis.

\subsection{Empirical modeling of X-ray outbursts}

In our sample, transients were observed basically during X-ray outbursts. The decay of the SXC is often composed of two distinct components (e.g., Woods et al. 2004): an initial quickly fading emission within $\sim 1 \mathrm{~d}$ and a longer time-scale one decaying on $\sim$ month. The former component, possibly related with burst activities, is beyond our present analyses. Below, we focus on the longer-decay component.

The absorbed SXC fluxes were already shown in Figure 7. which are in agreement with previous studies listed in Table 4. The light curves were derived in the following way over individual outbursts from the data described in $\$ 2.3$. Individual Swift and RXTE spectra were at first fitted by a single blackbody model (2.3), while the CBB model (2.1) was further employed to explain residuals in the higher energy band if the resultant reduced chi-square is not accepted $\left(\chi_{\nu}^{2}>1.4\right)$. All the spectral parameters were allowed to vary in each observation except the absorption column density $N_{\mathrm{H}}$ which is fixed at the value determined by high statistics observations from early phase observations with Suzaku/XIS or Swift/XRT. Below, we use flux measurements of acceptable fits, and detailed spectral characterization will be discussed elsewhere.

Figure 13 shows the $0.1-20 \mathrm{keV}$ luminosities of these outbursts after correcting for the photo-absorption (2.3) and distances $d$ (Appendix $(\underline{B}$ ). Such fading light curves in Figure 7 from different objects have so far been fitted by some empirical formulae: an exponential (Rea et al. 2009), double-exponential (Scholz et al. 2012), powerlaw (Lvubarsky et al. 2002; Kouveliotou et al. 2003) or broken power-law functions. Since there is no commonly established function applicable to all the outbursts, nor consensus on physical understanding of the decay, it is still meaningful to search for an empirical formula to uniformly investigate the outbursts.

The light curves imply that some transients exhibit a plateau-like constant period at the earliest phase, followed by a power-law decay, such as three transients: SGR 0501+4516, SGR 0418+5729, and Swift J1822.31606. In order to represent this behaviour, we employed a mathematical form as

$$
L(t)=\frac{L_{0}}{\left(1+t / \tau_{0}\right)^{p}} .
$$

Hereafter we call this Eq. (12) the plateau-decaying (PD) function since it becomes constant $L_{0}$ at $t \ll \tau_{0}$, while it becomes a power-law with an index of $p$ at $t \gg \tau_{0}$. For sources which plateau is not clear, we just used the power-law $(\mathrm{PL})$ model, $L_{\mathrm{x}}=L_{0}\left(t / \tau_{0}\right)^{-p}$ which is normalized at $\tau_{0}=1 \mathrm{~d}$. The resultant parameters are summarized in Table 4, and further comparison among difference fittings are described in Appendix C. As described there, the plateau is not an artefact of the definition of the time origin. The plateau period are $\tau_{0} \sim 11-43 \mathrm{~d}$ for the above three sources, and subsequent decay indices are $p \sim 0.7-2$. Other sources (e.g., 1E 1547.0-5408 and Swift J1834.9-0846) faded with a flatter index $p \sim 0.1-0.3$ without such a clear plateau duration.

The total energy $E_{\text {total }}$ released during a single outburst, an integration of Eq. (12), becomes $E_{\text {total }}=$ $\tau_{0} L_{0} /(p-1)$, in a steep decay case $(p>1)$, while that in the flatter decay is calculated with assuming an integration upper bound at $100 \mathrm{~d}$ as a typical decaying duration. Even under present uncertainties of distance measurements, as listed in Table [7 a typical $E_{\text {tot }}$ is a few of $10^{41}$ erg.

\section{DISCUSSION}

We compiled all archival Suzaku spectra of fifteen AXP and SGR sources acquired in 2006-2013, adding 10 new observations to Paper I. We also incorporated the Swift/XRT, RXTE/PCA, and NuSTAR public data. These data sets reinforced the magnetar hypothesis for SGRs and AXPs.

\subsection{Uniqueness of the two spectral components}

The characteristic spectral shapes, composed of the SXC $(\lesssim 10 \mathrm{keV})$ and $\mathrm{HXC}(\gtrsim 10 \mathrm{keV})$, have been revealed to be a common feature of this class. Figure 14. an expanded $P-\dot{P}$ plot, summarizes the currently available HXC information of all AXPs and SGRs. Combining INTEGRAL, RXTE, Suzaku (with 7 detections), and $N u S T A R$ which afforded the HXC detections from 1E 2259+586 (Vogel et al. 2014) and SGR J1745-29 near Sgr A* (Mori et al. 2013; Kaspi et al. 2014), the $\mathrm{HXC}$ has been confirmed from 9 objects among $\sim 23$ confirmed sources, and the detected 15-60 keV HXC flux level is in the range of $\sim 0.3-11 \times 10^{-11} \mathrm{ergs}^{-1} \mathrm{~cm}^{-2}$. 

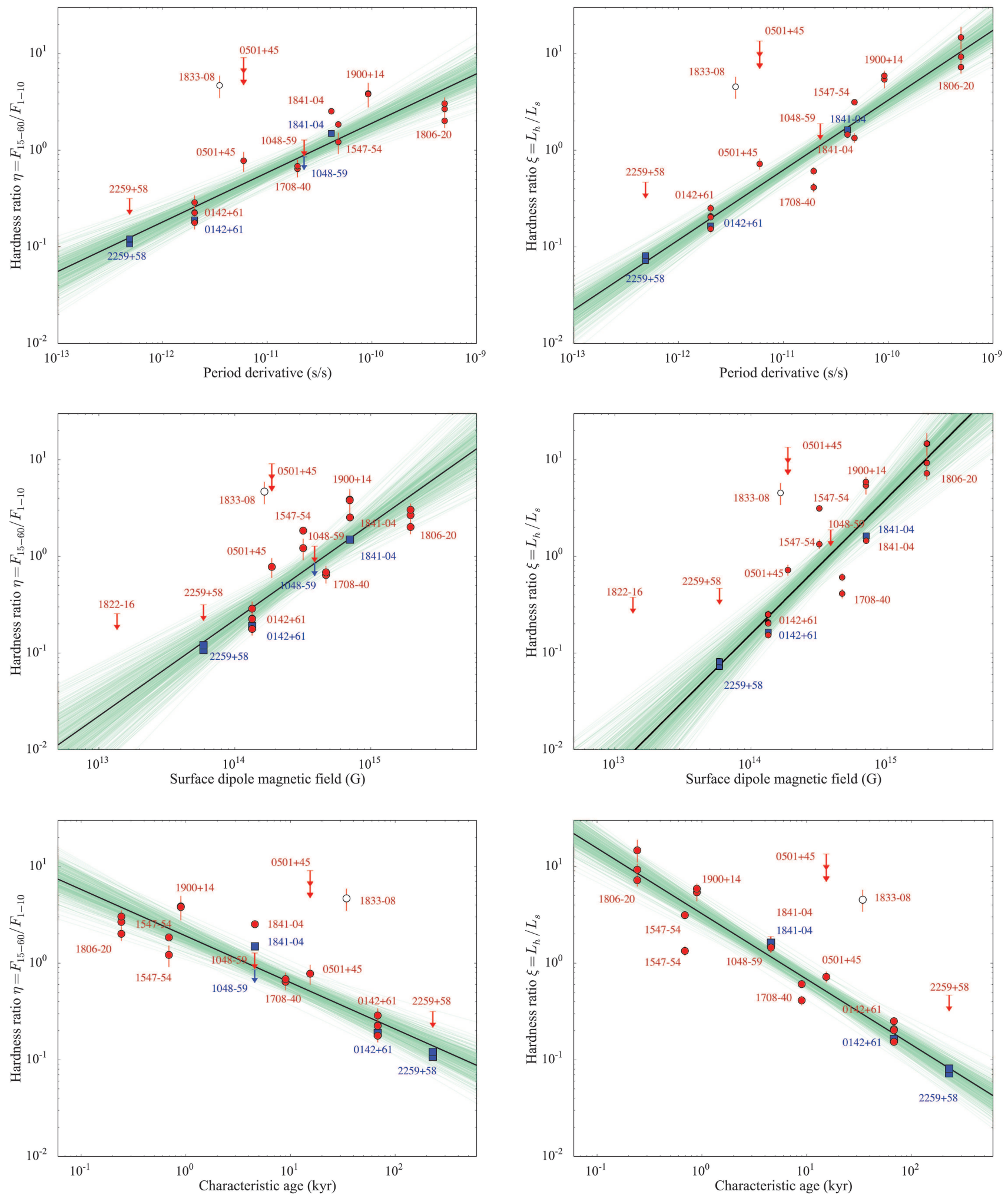

Figure 10. Hardness ratios of the HXC to the SXC (Table 6), defined using the absorbed fluxes $\eta=F_{15-60} / F_{1-10}$ (left panels) or using the unabsorbed luminosities $\eta=L_{\mathrm{h}} / L_{\mathrm{s}}$ (right panels), as a function of $\dot{P}$ (top panels). $B_{\mathrm{d}}$ (middle), and $\tau_{\mathrm{c}}$ (bottom). Filled circles and triangles are the data from Suzaku and NuSTAR, respectively. All the upper limits (arrows) are also indicated. The solid black line is the best fit regression model calculated in the linmix package (Kelly 2007). Green lines are samples from the corresponding posterior distribution of the model parameters. 

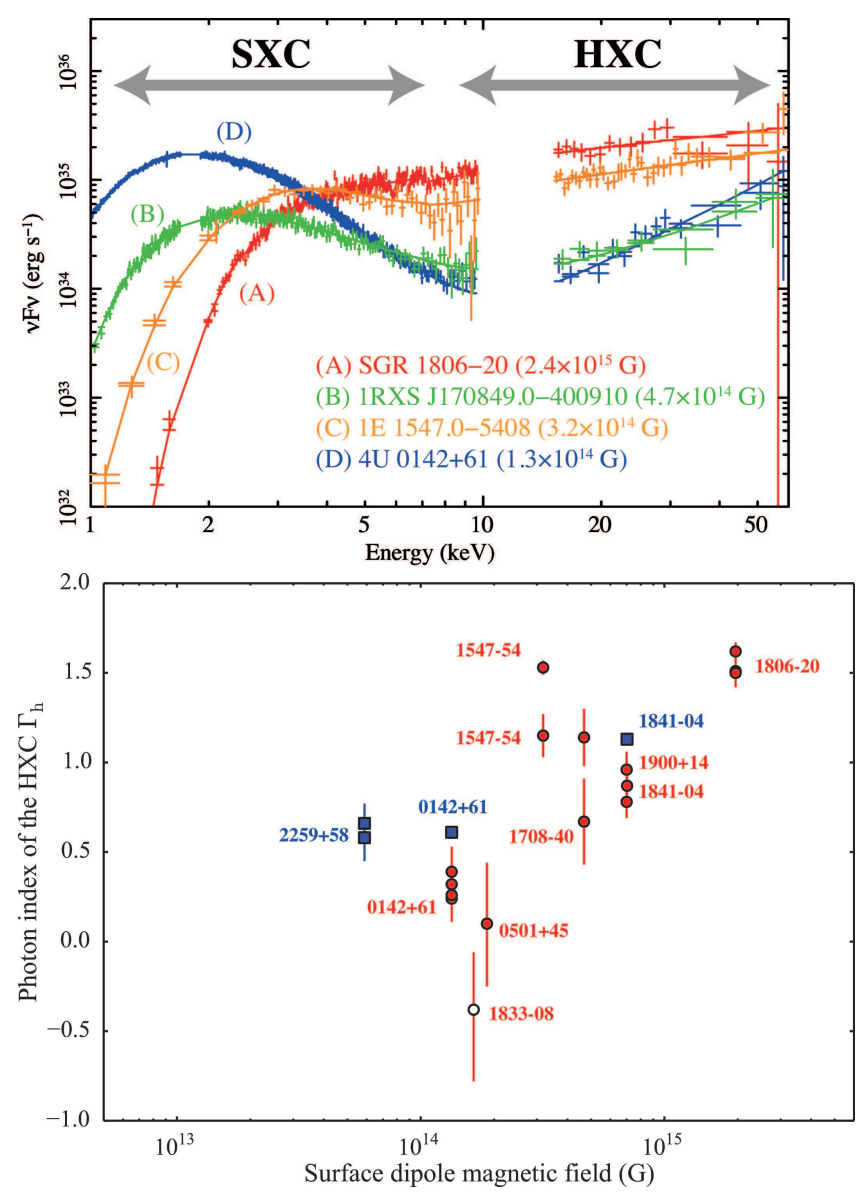

Figure 11. (top) Comparison of representative X-ray spectra of magnetars after correcting the data for their distances. (bottom) The photon index $\Gamma_{\mathrm{h}}$ of the HXC as a function of the magnetic field $B_{\mathrm{d}}$. Symbols are the same as in Figure 10

One important property of the HXC is that it exhibits the clear scalings, in terms of its luminosity ratio $\xi(3.1$ Fig 10, Eq,2, 5, and 6) and its spectral slope $\Gamma_{\mathrm{h}}(\mathrm{3.2.2}$. Fig[11k; Eq[7]. Since the scalings apply both to AXPs and SGRs, they can be collectively called magnetars at least from the view point of wide-band spectrum. These relations also allow us to predict the HXC intensity of a source, when its $P, \dot{P}$, and the SXC intensity are given. At 3.2 .1 we pointed out the narrow $P$ clustering of magnetars, and showed the $\eta$ and $\xi$ correlations to $\dot{P}$. In future, if young and fast rotating magnetars (e.g., $P \sim$ 0.1 s at $\sim 10-100 \mathrm{yr}$, as an origin of fast radio bursts, e.g., see Beloborodov 2017), or hypothetical slowly rotating magnetars (e.g., $P \sim 100$ s, as related with gamma-ray bursts Rea et al. 2015), would be discovered, the wider range of $P$ will provide another hint to judge a main control parameter of the spectral shape.

Do we observe such two-component spectra from other classes of magnetized neutron stars? Some rotationpowered pulsars, such as Vela pulsar or Geminga, exhibit two-component spectrum, consisting of thermal and nonthermal components. However, they have lower $k T_{\mathrm{s}}$ and steeper $\Gamma_{\mathrm{h}} \sim 1.5-2.5$ than those of the SGRs and AXPs, and show no clear scaling of $\xi-B_{\mathrm{d}}$. Accretion-powered X-ray pulsars emit predominantly in a single hard component (at least in $>2 \mathrm{keV}$ ), regardless of their luminos-

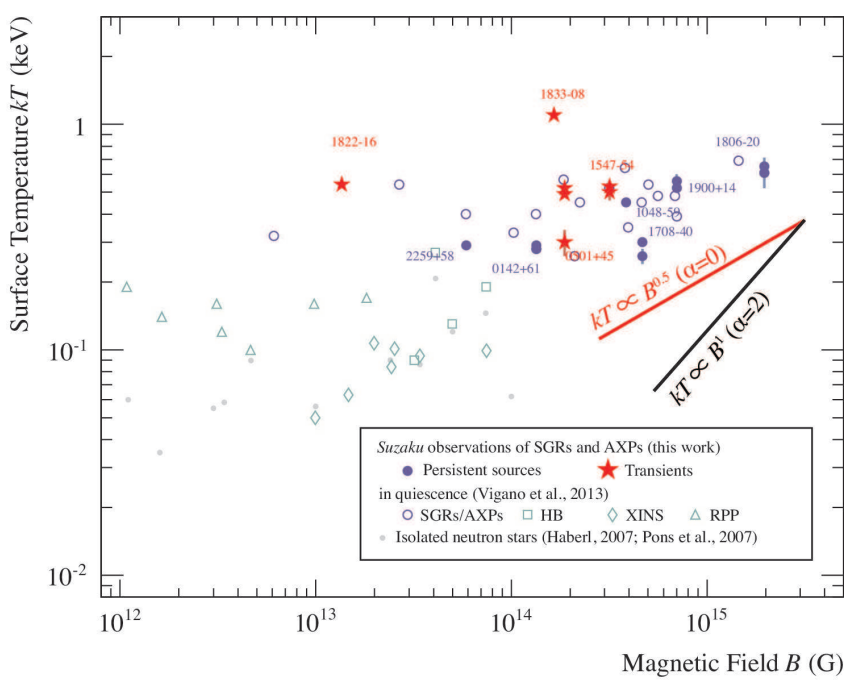

Figure 12. Surface temperature of magnetars measured with Suzaku in the CBB model (filled symbols) as a function of the surface dipole magnetic field $B_{\mathrm{d}}$. For comparison, isolated neutron stars in quiescence are shown (open symbols) from Viganò et al. (2013); SGRs /AXPs (open circles), high-B pulsars (open squares), $\mathrm{X}$-ray isolated neutron stars (open diamonds), and rotation powered pulsars (open triangles). Data from Haberl (2007); Pons et al. $(2007)$ are also compared. Two slopes are indicated with $T \propto B^{1}$ $(\alpha=2)$ and $k T \propto B^{0.5}(\alpha=0$, exponential decay of magnetic field)



Figure 13. The same as Figure 7 but the observed flux was converted to the unabsorbed $1-20 \mathrm{keV}$ luminosity, and the outburst decay was fitted with eq. (12).

ity (e.g., Terada et al. 2006). Thus, the two-component spectral composition is considered to be specific to magnetars.

\subsection{The reality of $B_{\mathrm{d}}$}

Different from the magnetar model, an alternative scenario to explain SGRs and AXPs assumes that $B_{\mathrm{d}}$ calculated from $P$ and $\dot{P}$ does not represent the true dipole field strength near a stellar surface nor exceed the critical field $B_{\mathrm{QED}}$. In this case, X-ray radiation is not powered from the magnetic energy but from accretion of a fallback disk which is left over from a supernova explosion of the progenitor Chatteriee et al. 2000; Alpar 2001b; Benli et al. 2013). The bulk-motion Comptonization of the accretion column is attributed to the $\mathrm{HXC}$ radiation 


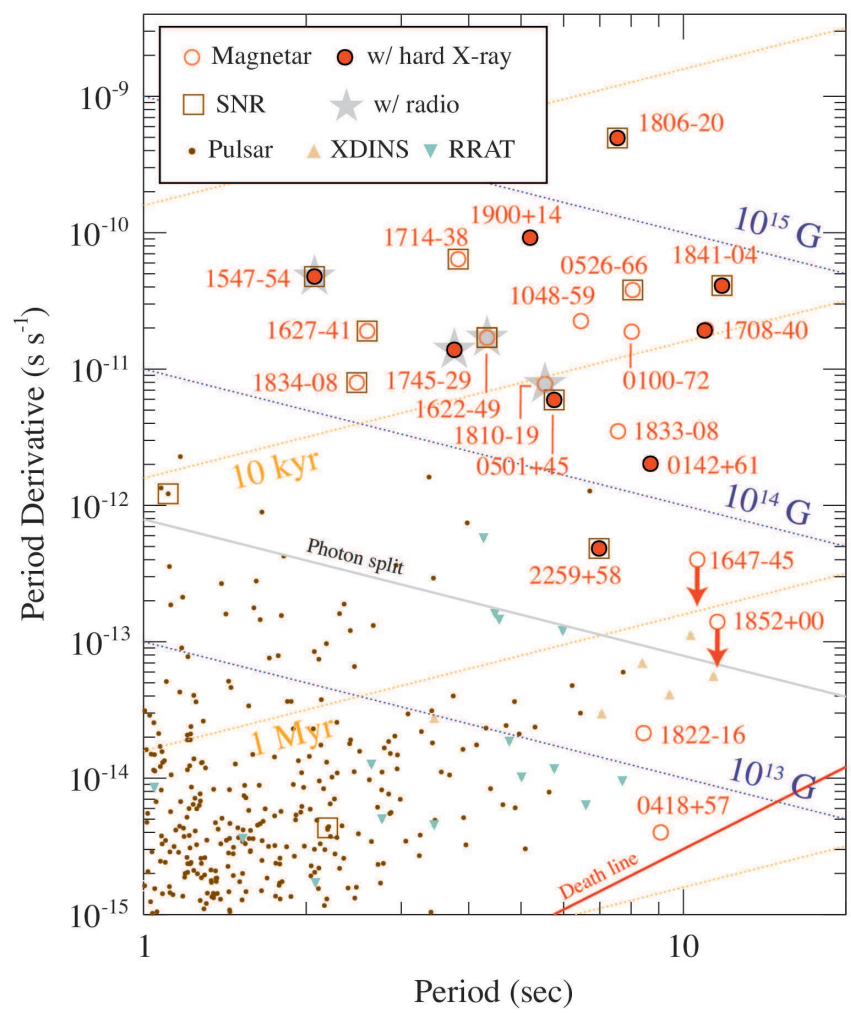

Figure 14. Magnetars (circle symbols) on the $P-\dot{P}$ diagram (zoom-up from Figure 21). Sources are shown in the filled symbols if the HXC are detected either by Suzaku, INTEGRAL, or $N u S$ $T A R$. Association with an SNR and detection in the radio band are shown in square and star marks, respectively. Canonical pulsars (dot symbols), X-ray Dim Isolated Neutron Stars (XDINS; triangles), and Rotating RAdio Transient (RRAT; inverted triangles) are shown for comparison.

\section{(Trümper et al. 2010, 2013; Kvlafis et al. 2014).}

The accretion scenario has still difficulties to explain the broad-band X-ray observations: 1) Firstly, as already stated above, the accretion-powered pulsars do not show distinct two spectral components even in the low X-ray luminosity. 2) The power-law HXC extend up to $\sim 100 \mathrm{keV}$ with the hard $\Gamma_{\mathrm{h}}$ without any absorption nor cutoff features which are usually expected from the electron cyclotron resonance of the ordinary accretionpowered neutron stars. 3) Finally, the spectral scalings implies that the $B_{\mathrm{d}}$ values play an important role for the emission mechanism, especially to the HXC. The nominal value $B_{\mathrm{d}}$ derived from $P$ and $\dot{P}$ is, therefore, considered to be a true poloidal component near the stellar surface.

The present work suggested that $B_{\mathrm{d}}$ is one main control parameter of the HXC radiation. Some theoretically motivated emission models have been already developed so far in the magnetar scheme; these include thermal bremsstrahlung (Thompson \& Beloborodov 2005), synchrotron radiation (Thompson \& Beloborodov 2005; Hevl \& Hernquist 2005), resonant scattering (Baring \& Harding 2007; Nobili et al. 2008; Viganò et al. 2012; Beloborodov 2013b), and downcascade due to the photon splitting (Paper I). Although the mechanism is still observationally poorly understood, the appropriate radiation scenario would include the physics in the strong magnetic field to explain the scaling. For example, the hard photon index $\Gamma_{\mathrm{h}}$ in our sample is correlated with $B_{\mathrm{d}}$. This can not be explained only by the difference of viewing angles, and should be further compared with models (e.g., Beloborodov 2013b).

One potential clue is how $\xi$ or $\Gamma_{\mathrm{h}}$ behave on the scaling plots during the outburst. For example, there is a signature of a faster decay of the HXC than the SXC in some sources: e.g., SGR 0501+4516, (Rea et al. 2009; Enoto et al. 2010c), and 1E 1547.0-5408 (Enoto et al., in prep.). This question has not yet to be clearly answered in the present Suzaku data. In addition, our current observations of the $\mathrm{HXC}$ are limited up to $\sim 60 \mathrm{keV}$. The HXC cutoff is expected at $\sim 400 \mathrm{keV}$ by the fossil disk model (Kylafis et al. 2014), while the annihilation line is suggested in some magnetar models (Beloborodov 2013b). Thus, soft gamma-ray observation is expected to provide a smoking gun.

\subsection{Implications of the $L_{\mathrm{x}}-L_{\mathrm{sd}}$ diagram}

In Figure [15] and Table [6, we compare the total Xray luminosity $L_{\mathrm{x}}=L_{\mathrm{h}}+L_{\mathrm{s}}$ (3) with the spin-down powers $L_{\mathrm{sd}}$. The $2-10 \mathrm{keV}$ luminosities of 41 ordinary rotation-powered pulsars follow a linear empirical relation, $\log L_{\mathrm{x}}=1.34 \log L_{\mathrm{sd}}-15.34$ (Possenti et al. 2002). They are well below the critical luminosity $\log L_{\text {crit }}=$ $1.48 \log L_{\mathrm{sd}}-18.5$, which is thought to be the maximum efficiency of conversion of the spin-down luminosity to X-ray emission. Our inclusion of $L_{\mathrm{h}}$ improved $L_{\mathrm{x}}$ measurements, and more clearly show the luminosity excess $\left(L_{\mathrm{x}} \gg L_{\mathrm{sd}}\right)$. Furthermore, $L_{\mathrm{x}}$ values exhibit little dependence on $L_{\mathrm{sd}}$. These properties have indeed been providing strong support to the magnetar hypothesis.

Highly variable transients mostly reach $\sim 10^{35} \mathrm{erg} \mathrm{s}^{-1}$ at an early stage of their outbursts and gradually decay back to quiescence below $\lesssim 10^{33} \mathrm{erg} \mathrm{s}^{-1}$. Some of them (e.g., 1E 1547.0-5408 and Swift J1834.9-0846) decay back to $L_{\mathrm{x}}<L_{\mathrm{sd}}$, to a region on Figure 14 which is adjacent to radio-loud normal rotation-powered pulsars (Rea et al. 2012a). However, it is still unclear whether AXPs and SGRs in their deep quiescence, can become dimmer than $L_{\text {crit }}$.

\subsection{Maximum luminosity and decay law of transients}

On the $L_{\mathrm{x}}-L_{\mathrm{sd}}$ diagram in Figure 15, the persistent Xray emission do not exceed $\sim 10^{36} \mathrm{erg} \mathrm{s}^{-1}$ either in persistent sources or in transients during an early phase of outbursts. In the light curves (\$3.3), transients sometimes show an initial plateau phase for $\sim 10-40 \mathrm{~d}$ keeping $L_{\mathrm{s}} \sim$ $10^{35}-10^{36} \mathrm{ergs}^{-1}$, in the same $L_{\mathrm{s}}$ range as the persistently sources. If the emission is a pure blackbody radiation, this typically corresponds to $L_{\max }=4 \pi R_{\text {spot }}^{2} \sigma T^{4}=$ $1.3 \times 10^{35}\left(R_{\text {spot }} / 1 \mathrm{~km}\right)^{2}(k T / 1 \mathrm{keV})^{4} \mathrm{ergs} \mathrm{s}^{-1}$. Such a ceiling X-ray luminosity could be interpreted as regulation via a temperature-sensitive neutrino cooling, if the magnetic energy is converted to thermal energy in the stellar crust (Potekhin et al. 2007; Rea et al. 2013b; Pons \& Rea 2012b; Esposito et al. 2013b).

As shown in Figure 16, outbursts of higher- $B_{\mathrm{d}}$ magnetars show more prolonged decay than those of lower- $B_{\mathrm{d}}$ sources. This supports the basic concept that outbursts from magnetars are powered by sudden dissipation of the magnetic energy, although we do not yet know whether 


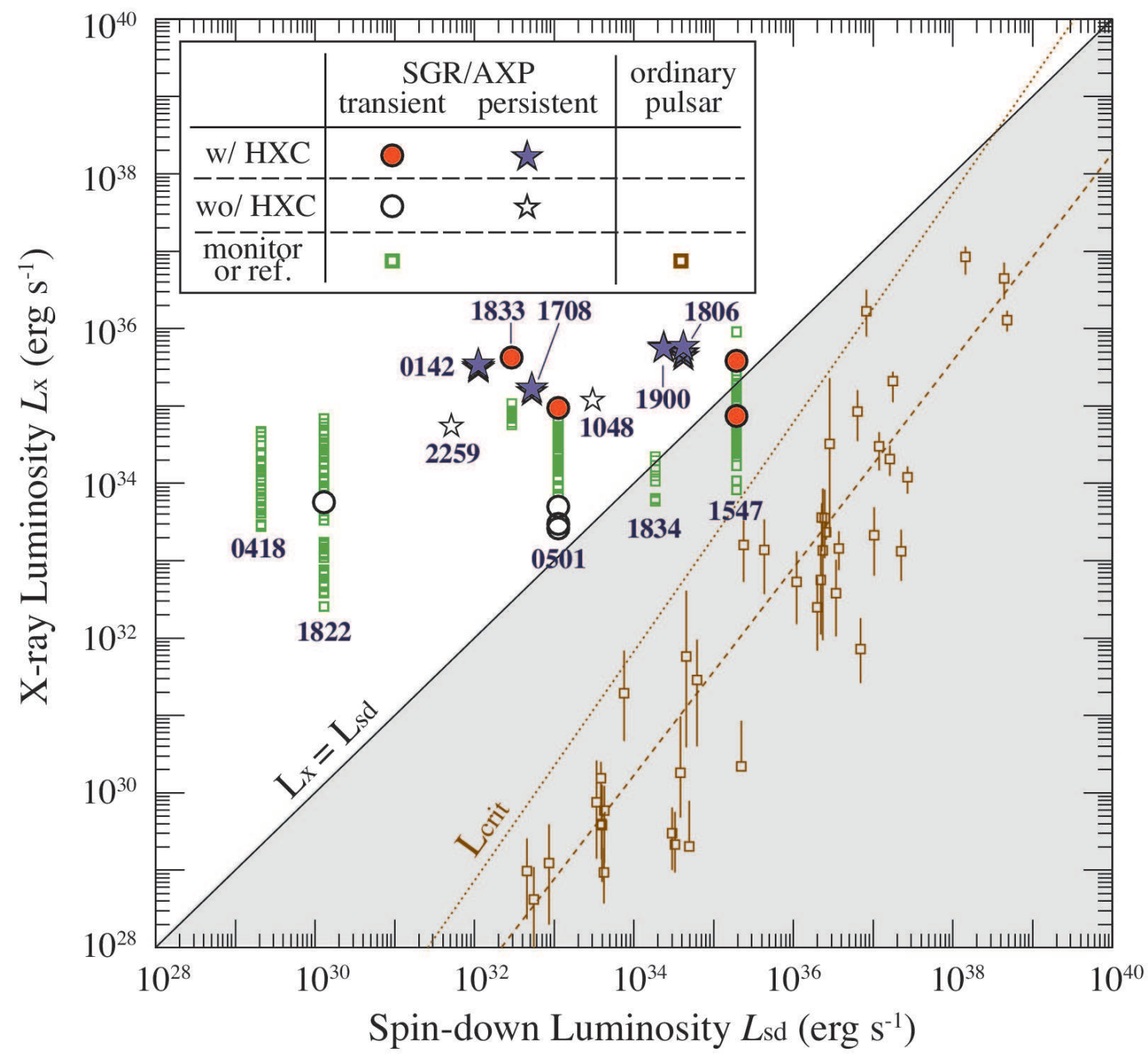

Figure 15. Observed 1-60 keV X-ray luminosity $L_{\mathrm{x}}=L_{\mathrm{s}}+L_{\mathrm{h}}(\mathrm{SXC}+\mathrm{HXC})$ of the objects listed in Table 6] compared with their spin-down luminosity $L_{\mathrm{sd}}$. Filled stars and circles indicate the persistently bright objects and transient ones, respectively. The sources are shown in filled symbols if the HXC are detected with Suzaku, while the others are open ones. The decaying X-ray luminosity monitored with Swift and RXTE are shown with small green squares. Ordinary rotation powered pulsars are shown in brown squares from Table 1 in Possenti et al. (2002). The employed distances and periods refer to Appendix B and the McGill catalog (Olausen \& Kaspi 2013). 


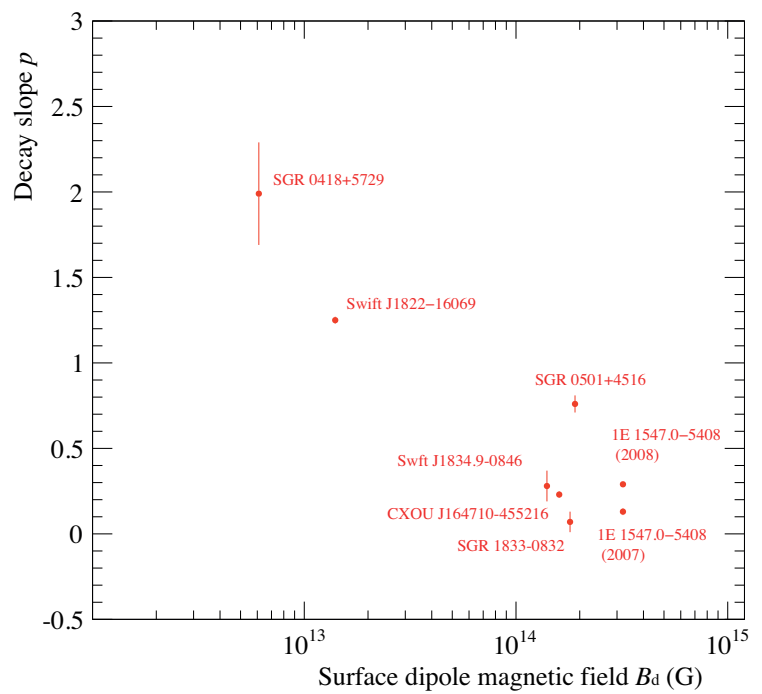

Figure 16. The decay slope $p$ (Eq. 12) shown as a function of $B_{\mathrm{d}}$.

the release occurs in the stellar interior Pons \& Rea 2012b; Li \& Beloborodov 2015) or in the magnetosphere (Beloborodov \& Thompson 2007; Kojima \& Kato 2014; Benli et al. 2015), or both.

The total emitted energy during a single X-ray outburst is typically $\sim 10^{41}-10^{42} \mathrm{erg}$. If this outburst can be attributed to the magnetic energy stored in the magnetar crust with a thickness of $\triangle R$ and a hot spot radius of $R_{\text {spot }} \sim 1 \mathrm{~km}$, i.e., $E_{\text {mag }}=\pi R_{\text {spot }}^{2} \triangle R \cdot B^{2} / 8 \pi=$ $2.4 \times 10^{41}\left(B / B_{\mathrm{QED}}\right)^{2}\left(R_{\text {spot }} / 1 \mathrm{~km}\right)^{2}(\triangle R / 1 \mathrm{~km}) \mathrm{erg}$, the required $\triangle R \sim 1 \mathrm{~km}$ is comparable to the crust thickness. Since low- $B_{\mathrm{d}}$ sources below $B_{\mathrm{QED}}$ (SGR $0418+5729$ and Swift J1822.3-1606) also radiate a similar amount of energy to those of other high- $B_{\mathrm{d}}$ sources, the actual surface field of low- $B_{\mathrm{d}}$ transients would be much stronger than estimated from $P-\dot{P}$ method.

Incidentally, the fall-back disk model tries to explain the outbursts in terms of sudden mass accretion Alpar 2001a; Benli et al. 2013, 2015). However, in such cases, the luminosity would hit a ceiling at the Eddington limit $\left(\sim 10^{38} \mathrm{erg} \mathrm{s}^{-1}\right)$ rather than at $\sim 10^{36} \mathrm{erg} \mathrm{s}^{-1}$; the outburst would decay exponentially (Tanaka \& Shibazaki 1996), or sometimes falls abruptly via the propeller effect (Asai et al. 2013) rather than in power-law form; and the spectrum around the outburst peak would exhibit clear fluorescence Fe-K line (e.g., EW>100 eV), if the fast initial rise of $\mathrm{X}$-ray outbursts indicates a mass reservoir close to the neutron star. Therefore, the scenario falls to account for several important properties of the actually observed outburst of magnetars.

\subsection{Speculative relation to seismology}

In the magnetar scenario, fading X-ray radiation of outbursts are usually theoretically modeled as a transient thermal response to a sudden energy release inside the stars. Originally proposed for post-glitch afterglows of ordinary neutron stars (Eichler \& Cheng 1989; Hirano et al. 1997), this idea is recently investigated in the magnetar context (Lyubarsky et al. 2002) and applied to X-ray observations of transient sources (Rea et al. 2012b; Scholz et al. 2012).
Instead of a large delta-function energy release assumed in previous models, we may alternatively assume that many small subsequent energy deposits take place during the X-ray afterglow as small starquakes or reconnections. Such a scheme remind us of seismology, where the occurrence frequency of aftershock sequence $n(t)$ after a large earthquake is known to follow an empirical formula, called the modified Omori's law, as $n(t) \propto(t+c)^{-p}$ (Utsu 1961). In seismology, $t$ is the elapsed time after the mainshock, $p \sim 0.7-1.5$ is the slope index of decay, and $c \lesssim 1 \mathrm{~d}$ is an empirical plateau phase duration (Utsu et al. 1995). This formula, thought to represent an underlying relaxation process after a mainshock, has the same mathematical form as the plateau-decaying function we used for X-ray outbursts (Eq. 9] in \$2.3).

We already know another statistical similarity of seismology to magnetars, i.e., Gutenberg-Richter law (Ishimoto \& Iida 1938; Gutenberg \& Richter 1941) seen in SGR short bursts: the number-intensity relations of their short burst fluence follows a power-law distribution (Götz et al. 2006; Nakagawa et al. 2007) as commonly seen in earthquakes and solar flares. This statistical relation is believed to represent self-organized criticality of the system (Aschwanden 2011). If, furthermore, the magnetar persistent emission (or a part of it) is composed of small energy releases, the plateau-decaying light curve is understood as changes in the normalization of the Gutenberg-Richter law during the outbursts (Nakagawa et al. 2009), providing another similarity of underlying physical process to seismology, where subsequent energy dissipation triggered by a previous event.

\section{CONCLUSION}

Our Suzaku observations of the SGR and AXP classes, combined with $N u S T A R$, Swift, and RXTE data, have yielded the following results. The magnetar interpretation have been strengthened from several aspects.

1. The HXC, $\sim 0.3-11 \times 10^{-11} \mathrm{erg} \mathrm{s}^{-1} \mathrm{~cm}^{-2}$ in the $15-$ $60 \mathrm{keV}$ band, is thought to be a common feature of the this class.

2. The scalings of $\xi$ and $\Gamma_{\mathrm{h}}$ found in Paper I (Eq. 2 [5] and (6), have been reinforced. $B_{\mathrm{d}}$ derived from $P$ and $\dot{P}$ is a key control parameter of the spectral evolution.

3. After including the HXC, differences from the ordinary rotation- or accretion-powered pulsars become more prominent: e.g., $L_{\mathrm{x}}>L_{\mathrm{sd}}$ and the ceiling luminosity at $10^{35-36} \mathrm{erg} \mathrm{s}^{-1}$.

4. The fading $L_{\mathrm{s}}$ during outbursts is approximated by a mathematical form of Eq. 12, implying a similarity to seismology.

The authors would like to express their thanks to the Suzaku team for their prompt ToO observations, and to Prof. Hiroyuki Nakanishi for his providing the hydrogen column density map of our Galaxy. This work was supported by JSPS KAKENHI Grant Numbers, 12J03320, 15H00845, 15H03653, 16H00869, 16H02198, 16K17665, 16J06773, 25105507, 25400221, and Hakubi project at 
Kyoto University. This research was also supported by the Munich Institute for Astro- and Particle Physics (MIAPP) of the DFG cluster of excellence "Origin and Structure of the Universe."

\section{REFERENCES}

Alpar, M. A. 2001a, ApJ, 554, 1245

-. 2001b, ApJ, 554, 1245

An, H., Hascoët, R., Kaspi, V. M., et al. 2013a, ApJ, 779, 163 —. 2013b, ApJ, 779, 163

An, H., Kaspi, V. M., Beloborodov, A. M., et al. 2014a, ApJ, 790, 60

An, H., Kaspi, V. M., Archibald, R., et al. 2014b, Astronomische Nachrichten, 335, 280

Anders, E., \& Grevesse, N. 1989, Geochim. Cosmochim. Acta, 53, 197

Aptekar, R. L., Cline, T. L., Frederiks, D. D., et al. 2009, ApJ, 698, L82

Asai, K., Matsuoka, M., Mihara, T., et al. 2013, ApJ, 773, 117

Aschwanden, M. J. 2011, Self-Organized Criticality in Astrophysics

Balucinska-Church, M., \& McCammon, D. 1992, ApJ, 400, 699

Baring, M. G., \& Harding, A. K. 1998, ApJ, 507, L55

-. 2007, Ap\&SS, 308, 109

Barthelmy, S. D., Barbier, L. M., Cummings, J. R., et al. 2005, Space Sci. Rev., 120, 143

Beloborodov, A. M. 2013a, ApJ, 762, 13

-. 2013b, ApJ, 762, 13

-. 2017, ArXiv e-prints, arXiv:1702.08644

Beloborodov, A. M., \& Thompson, C. 2007, ApJ, 657, 967

Benli, O., Çalışkan, Ş., Ertan, Ü., et al. 2013, ApJ, 778, 119

Benli, O., SCalskan, S., \& Ertan, Ü. 2015, MNRAS, 447, 2282

Bibby, J. L., Crowther, P. A., Furness, J. P., \& Clark, J. S. 2008, MNRAS, 386, L23

Bradt, H. V., Rothschild, R. E., \& Swank, J. H. 1993, A\&AS, 97, 355

Burrows, D. N., Hill, J. E., Nousek, J. A., et al. 2005, Space Sci. Rev., 120, 165

Camilo, F., Ransom, S. M., Halpern, J. P., \& Reynolds, J. 2007, ApJ, 666, L93

Chatterjee, P., Hernquist, L., \& Narayan, R. 2000, ApJ, 534, 373

Chen, K., \& Ruderman, M. 1993, ApJ, 402, 264

Clark, J. S., Negueruela, I., Crowther, P. A., \& Goodwin, S. P. 2005, A\&A, 434, 949

Colpi, M., Geppert, U., \& Page, D. 2000, ApJ, 529, L29

Cummings, J. R., Burrows, D., Campana, S., et al. 2011, The Astronomer's Telegram, 3488, 1

Dall'Osso, S., Granot, J., \& Piran, T. 2012, MNRAS, 422, 2878

Davies, B., Figer, D. F., Kudritzki, R.-P., et al. 2009, ApJ, 707, 844

D'Elia, V., Barthelmy, S. D., Baumgartner, W. H., et al. 2011, GRB Coordinates Network, 12253, 1

den Hartog, P. R., Kuiper, L., \& Hermsen, W. 2008a, A\&A, 489, 263

den Hartog, P. R., Kuiper, L., Hermsen, W., et al. 2008b, A\&A, 489, 245

Dib, R., \& Kaspi, V. M. 2014, ApJ, 784, 37

Duncan, R. C., \& Thompson, C. 1992, ApJ, 392, L9

Durant, M., \& van Kerkwijk, M. H. 2006, ApJ, 650, 1070

Eichler, D., \& Cheng, A. F. 1989, ApJ, 336, 360

Enoto, T., Makishima, K., Nakazawa, K., et al. 2011, PASJ, 63, 387

Enoto, T., Nakagawa, Y. E., Sakamoto, T., \& Makishima, K. 2012, MNRAS, 427, 2824

Enoto, T., Nakazawa, K., Makishima, K., et al. 2010a, ApJ, 722, L162

Enoto, T., Nakagawa, Y. E., Rea, N., et al. 2009, ApJ, 693, L122

Enoto, T., Nakazawa, K., Makishima, K., et al. 2010b, PASJ, 62, 475

Enoto, T., Rea, N., Nakagawa, Y. E., et al. 2010c, ApJ, 715, 665

Esposito, P., Mereghetti, S., Tiengo, A., et al. 2007, A\&A, 476, 321

Esposito, P., Israel, G. L., Turolla, R., et al. 2010, MNRAS, 405, 1787
-. 2011, MNRAS, 416, 205

Esposito, P., Tiengo, A., Rea, N., et al. 2013a, MNRAS, 429, 3123

-. 2013b, MNRAS, 429, 3123

Fukazawa, Y., Mizuno, T., Watanabe, S., et al. 2009, PASJ, 61, 17

Gaensler, B. M., \& Chatterjee, S. 2008, GRB Coordinates

Network, 8149, 1

Gavriil, F. P., Kaspi, V. M., \& Woods, P. M. 2002, Nature, 419, 142

Gehrels, N., Chincarini, G., Giommi, P., et al. 2004, ApJ, 611, 1005

Gelbord, J. M., Barthelmy, S. D., Baumgartner, W. H., et al. 2010, GRB Coordinates Network, 10526, 1

Gelfand, J. D., \& Gaensler, B. M. 2007, ApJ, 667, 1111

Goldreich, P., \& Reisenegger, A. 1992, ApJ, 395, 250

Götz, D., Mereghetti, S., Tiengo, A., \& Esposito, P. 2006, A\&A, 449, L31

Göğüss, E., Cusumano, G., Levan, A. J., et al. 2010, ApJ, 718, 331

Gronwall, C., Holland, S. T., Markwardt, C. B., et al. 2009, GRB

Coordinates Network, 8833, 1

Gutenberg, G., \& Richter, C. F. 1941, Geol. Soc. Am. Spec., 34, 1

Güver, T., Göğüs, E., \& Özel, F. 2011, MNRAS, 418, 2773

Haberl, F. 2007, Ap\&SS, 308, 181

Halpern, J. P., Gotthelf, E. V., Reynolds, J., Ransom, S. M., \& Camilo, F. 2008, ApJ, 676, 1178

Harding, A. K., \& Lai, D. 2006, Reports on Progress in Physics, 69,2631

Harrison, F. A., Craig, W. W., Christensen, F. E., et al. 2013, ApJ, 770, 103

Heyl, J. S., \& Hernquist, L. 2005, MNRAS, 362, 777

Hirano, S., Shibazaki, N., Umeda, H., \& Nomoto, K. 1997, ApJ, 491, 286

Holland, S. T., Barthelmy, S. D., Baumgartner, W. H., et al. 2008, GRB Coordinates Network, 8112, 1

Ishimoto, M., \& Iida, K. 1938, Bull. Earthq. Res. Inst. Univ. Tokyo, 17, 443

Ishisaki, Y., Maeda, Y., Fujimoto, R., et al. 2007, PASJ, 59, 113

Israel, G. L., Campana, S., Dall'Osso, S., et al. 2007, ApJ, 664, 448

Israel, G. L., Romano, P., Mangano, V., et al. 2008, ApJ, 685, 1114

Israel, G. L., Esposito, P., Rea, N., et al. 2010, MNRAS, 408, 1387

Iwahashi, T., Enoto, T., Yamada, S., et al. 2013, PASJ, 65, 52

Jahoda, K., Markwardt, C. B., Radeva, Y., et al. 2006, ApJS, 163,401

Kargaltsev, O., Kouveliotou, C., Pavlov, G. G., et al. 2012, ApJ, 748,26

Kaspi, V. M. 2007, Ap\&SS, 308, 1

Kaspi, V. M., \& Boydstun, K. 2010, ApJ, 710, L115

Kaspi, V. M., Gavriil, F. P., Woods, P. M., et al. 2003, ApJ, 588, L93

Kaspi, V. M., Archibald, R. F., Bhalerao, V., et al. 2014, ApJ, 786,84

Kelly, B. C. 2007, ApJ, 665, 1489

Kitaguchi, T., An, H., Beloborodov, A. M., et al. 2014, ApJ, 782, 3

Kojima, Y., \& Kato, Y. E. 2014, Progress of Theoretical and Experimental Physics, 2014, 023E01

Kokubun, M., Makishima, K., Takahashi, T., et al. 2007, PASJ, 59,53

Kothes, R., \& Dougherty, S. M. 2007, A\&A, 468, 993

Kothes, R., Uyaniker, B., \& Yar, A. 2002, ApJ, 576, 169

Kouveliotou, C., Dieters, S., Strohmayer, T., et al. 1998, Nature, 393,235

Kouveliotou, C., Eichler, D., Woods, P. M., et al. 2003, ApJ, 596, L79

Koyama, K., Tsunemi, H., Dotani, T., et al. 2007, PASJ, 59, 23

Krimm, H., Barthelmy, S., Campana, S., et al. 2006, GRB Coordinates Network, 5581, 1

Krimm, H. A., Beardmore, A. P., Burrows, D. N., et al. 2008, GRB Coordinates Network, 8311, 1

Krivonos, R., Revnivtsev, M., Churazov, E., et al. 2007, A\&A, 463, 957

Kuiper, L., Hermsen, W., den Hartog, P. R., \& Collmar, W. 2006, ApJ, 645, 556

Kuiper, L., Hermsen, W., den Hartog, P. R., \& Urama, J. O. 2012, ApJ, 748, 133 
Kumar, H. S., Safi-Harb, S., Slane, P. O., \& Gotthelf, E. V. 2014, ApJ, 781, 41

Kylafis, N. D., Trümper, J. E., \& Ertan, Ü. 2014, A\&A, 562, A62 Leahy, D. A., \& Tian, W. W. 2007, A\&A, 461, 1013

-. 2008, AJ, 135, 167

Li, X., \& Beloborodov, A. M. 2015, ApJ, 815, 25

Lin, L., Kouveliotou, C., Baring, M. G., et al. 2011, ApJ, 739, 87

Lyubarsky, Y., Eichler, D., \& Thompson, C. 2002, ApJ, 580, L69

Lyutikov, M. 2003, MNRAS, 346, 540

Lyutikov, M., \& Gavriil, F. P. 2006, MNRAS, 368, 690

Maeda, Y., Someya, K., Ishida, M., Hayashida, K., \& Mori, H. 2008, JX-ISAS-SUZAKU-MEMO-2008-06,

JX-ISAS-SUZAKU-MEMO-2008-06

Makishima, K., Enoto, T., Hiraga, J. S., et al. 2014, Physical Review Letters, 112, 171102

Makishima, K., Enoto, T., Murakami, H., et al. 2015, PASJ, arXiv:1501.07684

Malheiro, M., Rueda, J. A., \& Ruffini, R. 2012, PASJ, 64, 56

Manchester, R. N., Hobbs, G. B., Teoh, A., \& Hobbs, M. 2005, AJ, 129, 1993

Matsuoka, M., Kawasaki, K., Ueno, S., et al. 2009, PASJ, 61, 999

Mereghetti, S. 2008, A\&A Rev., 15, 225

Mereghetti, S., Esposito, P., Tiengo, A., et al. 2012, A\&A, 546, A30

Mereghetti, S., \& Stella, L. 1995, ApJ, 442, L17

Mitsuda, K., Bautz, M., Inoue, H., et al. 2007, PASJ, 59, 1

Moretti, A., Pagani, C., Cusumano, G., et al. 2009, A\&A, 493, 501

Mori, K., Gotthelf, E. V., Zhang, S., et al. 2013, ApJ, 770, L23

Morii, M., Sato, R., Kataoka, J., \& Kawai, N. 2003, PASJ, 55, L45

Morii, M., Kitamoto, S., Shibazaki, N., et al. 2010, PASJ, 62, 1249

Naik, S., Dotani, T., Kawai, N., et al. 2008, PASJ, 60, 237

Nakagawa, Y. E., Makishima, K., \& Enoto, T. 2011, PASJ, 63, 813

Nakagawa, Y. E., Yoshida, A., Yamaoka, K., \& Shibazaki, N. 2009, PASJ, 61, 109

Nakagawa, Y. E., Yoshida, A., Hurley, K., et al. 2007, PASJ, 59, 653

Nakamura, R., Bamba, A., Ishida, M., et al. 2009, PASJ, 61, 197

Nakanishi, H., \& Sofue, Y. 2003, PASJ, 55, 191

-. 2006, PASJ, 58, 847

Nakano, T., Murakami, H., Makishima, K., et al. 2015, PASJ, 67, 9

Ng, C.-Y., Kaspi, V. M., Dib, R., et al. 2011, ApJ, 729, 131

Nicholl, M., Smartt, S. J., Jerkstrand, A., et al. 2013, Nature, 502,346

Nielbock, M., Chini, R., Jütte, M., \& Manthey, E. 2001, A\&A, 377,273

Nobili, L., Turolla, R., \& Zane, S. 2008, MNRAS, 389, 989

Oates, S. R., Baumgartner, W. H., Beardmore, A. P., et al. 2011, GRB Coordinates Network, 12209, 1

Olausen, S. A., \& Kaspi, V. M. 2013, ArXiv e-prints, arXiv:1309.4167

—. 2014, ApJS, 212, 6

Ouyed, R., Elgarøy, Ø., Dahle, H., \& Keränen, P. 2004, A\&A, 420, 1025

Pons, J. A., Link, B., Miralles, J. A., \& Geppert, U. 2007, Physical Review Letters, 98, 071101

Pons, J. A., \& Rea, N. 2012a, ApJ, 750, L6

一. 2012b, ApJ, 750, L6

Possenti, A., Cerutti, R., Colpi, M., \& Mereghetti, S. 2002, A\&A, 387, 993

Potekhin, A. Y., Chabrier, G., \& Yakovlev, D. G. 2007, Ap\&SS, 308,353
Rea, N., \& Esposito, P. 2011, in High-Energy Emission from Pulsars and their Systems, ed. D. F. Torres \& N. Rea, 247 Rea, N., Gullón, M., Pons, J. A., et al. 2015, ApJ, 813, 92 Rea, N., Pons, J. A., Torres, D. F., \& Turolla, R. 2012a, ApJ, 748, L12

Rea, N., Zane, S., Turolla, R., Lyutikov, M., \& Götz, D. 2008, ApJ, 686, 1245

Rea, N., Israel, G. L., Turolla, R., et al. 2009, MNRAS, 396, 2419

Rea, N., Esposito, P., Turolla, R., et al. 2010, Science, 330, 944

Rea, N., Israel, G. L., Esposito, P., et al. 2012b, ApJ, 754, 27

Rea, N., Israel, G. L., Pons, J. A., et al. 2013a, ApJ, 770, 65

—. 2013b, ApJ, 770, 65

Rivera-Ingraham, A., \& van Kerkwijk, M. H. 2010, ApJ, 710, 797

Sato, T., Bamba, A., Nakamura, R., \& Ishida, M. 2010, PASJ, 62, L33

Scholz, P., Ng, C.-Y., Livingstone, M. A., et al. 2012, ApJ, 761, 66 Sugizaki, M., Mitsuda, K., Kaneda, H., et al. 2001, ApJS, 134, 77 Suwa, Y., \& Enoto, T. 2014, MNRAS, 443, 3586

Takahashi, T., Abe, K., Endo, M., et al. 2007, PASJ, 59, 35

Tanaka, Y., \& Shibazaki, N. 1996, ARA\&A, 34, 607

Tendulkar, S. P., Hascöet, R., Yang, C., et al. 2015, ApJ, 808, 32

Terada, Y., Mihara, T., Nakajima, M., et al. 2006, ApJ, 648, L139

Thompson, C., \& Beloborodov, A. M. 2005, ApJ, 634, 565

Thompson, C., \& Duncan, R. C. 1995, MNRAS, 275, 255

-. 1996, ApJ, 473, 322

Thompson, C., Lyutikov, M., \& Kulkarni, S. R. 2002, ApJ, 574, 332

Tian, W. W., \& Leahy, D. A. 2008, ApJ, 677, 292

Tian, W. W., Leahy, D. A., \& Li, D. 2010, MNRAS, 404, L1

Tiengo, A., Mereghetti, S., Turolla, R., et al. 2005, A\&A, 437, 997

Tiengo, A., Vianello, G., Esposito, P., et al. 2010, ApJ, 710, 227

Trümper, J. E., Dennerl, K., Kylafis, N. D., Ertan, Ü., \& Zezas, A. 2013, ApJ, 764, 49

Trümper, J. E., Zezas, A., Ertan, Ü., \& Kylafis, N. D. 2010, A\&A, 518, A46

Utsu, T. 1961, Geophys. Mag., 30, 521

Utsu, T., Ogata, Y., \& Matsu'ura, R. S. 1995, J. Phys. Earth, 43,

Valinia, A., \& Marshall, F. E. 1998, ApJ, 505, 134

van der Horst, A. J., Connaughton, V., Kouveliotou, C., et al. 2009, The Astronomer's Telegram, 2077, 1

—. 2010, ApJ, 711, L1

Viganò, D., Parkins, N., Zane, S., et al. 2012, Journal of Physics Conference Series, 342, 012013

Viganò, D., Rea, N., Pons, J. A., et al. 2013, MNRAS, 434, 123

Vink, J., \& Kuiper, L. 2006, MNRAS, 370, L14

Vogel, J. K., Hascoët, R., Kaspi, V. M., et al. 2014, ApJ, 789, 75

Weng, S.-S., \& Göğüs, E. 2015, ApJ, 815, 15

White, N. E., Angelini, L., Ebisawa, K., Tanaka, Y., \& Ghosh, P. 1996, ApJ, 463, L83

Wik, D. R., Hornstrup, A., Molendi, S., et al. 2014, ApJ, 792, 48

Woods, P. M., \& Thompson, C. 2006, Soft gamma repeaters and anomalous X-ray pulsars: magnetar candidates, ed. W. H. G. Lewin \& M. van der Klis, 547-586

Woods, P. M., Kaspi, V. M., Thompson, C., et al. 2004, ApJ, 605, 378

Yamada, S., Uchiyama, H., Dotani, T., et al. 2012, PASJ, 64, 53

Yamazaki, R. 2009, ApJ, 690, L118

Yang, C., Archibald, R. F., Vogel, J. K., et al. 2015, ArXiv e-prints, arXiv:1509.08115

Zane, S., Rea, N., Turolla, R., \& Nobili, L. 2009, MNRAS, 398, 1403

Zhang, B., Harding, A. K., \& Muslimov, A. G. 2000, ApJ, 531, L135 


\section{NOTE ON INDIVIDUAL SOURCES UPDATED FROM PAPER I}

Here we summarize updated information of individual observations from Paper I.

\section{SGR 1833-0832}

On 2010 March 19, SGR 1833-0832 was discovered from short bursts. A Suzaku ToO was performed on March 27 for an effective $40 \mathrm{ks}$ exposure, $\sim 8.4$ days after the discovery. The $0.5-10 \mathrm{keV}$ XIS light curve is stable without any clear short burst detections. The pulsation was detected with XIS0 (1/8 window mode) at $P=7.565 \pm 0.001 \mathrm{~s}$ at the MJD epoch of 55272. The pulsed fraction is $\mathrm{PF}=\left(F_{\max }-F_{\min }\right) /\left(F_{\max }+F_{\min }\right) \sim 60-80 \%(2-10 \mathrm{keV})$, where $F_{\max }$ and $F_{\min }$ are the background-subtracted maximum and minimum count rates of the pulse profile. When fitting the 1-10 $\mathrm{keV}$ XIS data alone, the SXC spectrum is fitted by a single blackbody of $k T=1.22 \mathrm{keV}$ with $N_{\mathrm{H}}=9.0 \times 10^{22} \mathrm{~cm}^{-2}$ or by a single power-law of $\Gamma=3.1$ with $N_{\mathrm{H}}=1.6 \times 10^{23} \mathrm{~cm}^{-2}$. The HXC detection is marginal (Table 2). Comparing with Earth occultation data, the simulated HXD-PIN NXB was found to be slightly under-estimated by $\sim 2 \%$ than other observations, and if we assigned this additional $2 \%$ to the NXB uncertainty, the HXC detection significance reduced to $2.4 \sigma$ level. Thus, we just regard the HXC of SGR 1833-0832 as a marginal signal.

$$
4 U 0142+61
$$

First and second Suzaku observations were performed in 2007 and 2009 and already reported focusing the spectral feature and pulse modulation, respectively (Enoto et al. 2011; Makishima et al. 2014). A $\sim 37 \mathrm{ks}$ ToO was performed in 2011 September after short burst activities on July 29, 2011 (Oates et al.|2011) which states showed a slight brightening than previous observations. Another $\sim 80 \mathrm{ks}$ AO8 observation in 2013 July is planned to report elsewhere.

\section{AX J1818.8-1559}

A magnetar candidate AX J1818.8-1559 was discovered from the ASCA Galactic survey (Sugizaki et al. 2001). Mereghetti et al. (2012) performed a Suzaku follow-up observation, combined with Chandra and XMM-Newton spectral studies, after a detection of a magnetar-like short burst with INTEGRAL in 2007. Due to low photon counts of the XIS spectra, we fixed $N_{\mathrm{H}}$ value within the error range of (Mereghetti et al. 2012), and the derived flux and $k T$ values are consistent with their report.

$$
1 E \text { 1841-045 (Kes 73) }
$$

An angular radius $\sim 2^{\prime}$ of the shell-type SNR Kes 73 is comparable with the HPD of the XRT. To take into account the SNR contamination into the XIS data, Morii et al. (2010) used the Chandra SNR observation (Morii et al. 2003) to fit the Suzaku spectrum, and we previously used the XMM result (Vink \& Kuiper 2006) in Paper I. In this paper, we replaced it to the latest study based on the combined Chandra and XMM analyses described in Table 3 by Kumar et al. (2014), where two soft and hard components, corresponding to the swept-up interstellar medium and ejecta, are represented by VPSHOCK +VPSHOCK models in XSPEC. Since there is spatial variation of the emission lines, we let the relative normalization of the SNR to the point source and intensities of emission lines free within errors described in (Kumar et al. 2014), while fixed the normalization between two SNR component.

\section{Swift J1822.3-1606}

The 8.4-s X-ray pulsar Swift J1822.3-1606 was discovered on 2011 July by Swift/BAT and observed by different Xray satellites (Cummings et al. 2011; Rea et al. 2012b; Scholz et al. 2012). The derived dipole field $B_{\mathrm{d}}=1.4 \times 10^{13} \mathrm{G}$ is weaker than the critical field $B_{\mathrm{QED}}$, and called a low-field magnetar. Suzaku observation was performed on September 13, nearly two month later after the discovery. The soft X-ray data was already reported in Rea et al. (2012b), and we report here the $15-60 \mathrm{keV}$ absorbed flux upper limit at $1.2 \times 10^{-11} \mathrm{erg} \mathrm{s}^{-1} \mathrm{~cm}^{-2}$.

\section{CXOU J171405.7-381031 (CTB37B)}

We reanalyzed the same Suzaku data in 2006 (ObsID 501007010) which Nakamura et al. (2009) already published. With a characterization of a supernova remnant CTB 37B, they suggested a central compact source CXOU J171405.7-381031 is an AXP from their Chandra and Suzaku spectroscopies. The source was soon identified as a magnetar from XMM-Newton detections of slow pulsation and its large derivative (Sato et al. 2010). Since our XIS spectra is contaminated from a thermal component of CTB 37B, we added a "vnei" model with parameters fixed at values described in Nakamura et al. (2009). Despite the difference of employed spectral models (i.e, Comptonized blackbody with the power-law vs. a pure power-law), our derived parameters of $\Gamma_{\mathrm{s}} \sim 3.3$ and $N_{\mathrm{H}} \sim 3.5 \times 10^{22} \mathrm{~cm}^{-2}$ are consistent within errors with Nakamura et al. (2009). The hard X-ray data is not analyzed in detail due to a potential contamination from a surrounding CTB 37B.

$$
\text { 1E 1048.1-5937 }
$$

The X-ray source 1E 1048.1-5937 was observed with Suzaku on 2008 November 30 with $\sim 85$ ks exposure. The SXC was clearly detected, but the HXC was not able to detected with its $3 \sigma$ upper limit at $1.3 \times 10^{-11} \mathrm{erg} \mathrm{s}^{-1} \mathrm{~cm}^{-2}$ in the 15-60 keV band. The HXC was not detected with NuSTAR either in 2013 July, while the SXC was detected up to $\sim 20 \mathrm{keV}$ (Yang et al. 2015; Weng \& Göğüs 2015). Yang et al. (2015) reported the 20-79 keV flux upper limit at $<4.15 \times 10^{-12} \mathrm{erg} \mathrm{s}^{-1} \mathrm{~cm}^{-2}(3 \sigma)$, which is converted to $F_{15-60}<6.7 \times 10^{-12} \mathrm{erg} \mathrm{s}^{-1} \mathrm{~cm}^{-2}$ in the $15-60 \mathrm{keV}$ band, assuming the spectral shape $\Gamma_{\mathrm{s}}=3.64$ in Yang et al. (2015). Combining with $F_{1-10}=8.9 \times 10^{-12} \mathrm{erg} \mathrm{s}^{-1} \mathrm{~cm}^{-2}$, $\eta<0.75$. Since the true $\Gamma_{\mathrm{h}}$ is not known, we did not use $F_{\mathrm{h}}$ and $\xi$ values. 
Table 7

Distances $d$ to known SGRs and AXPs used in this paper.

\begin{tabular}{lrl}
\hline \hline \multicolumn{1}{c}{ Source name } & $\begin{array}{c}d \\
(\mathrm{kpc})\end{array}$ & \multicolumn{1}{c}{ Assumption or Method (Reference) } \\
\hline SGR 1806-20 & $8.7_{-1.5}^{+1.8}$ & host cluster 1806-20 (G10.0-0.3) (Bibby et al. 2008) \\
1E 1841-045 & $8.5_{-1.0}^{+1.3}$ & association to SNR Kes 73 (Tian \& Leahy 2008) \\
SGR 1900+14 & $12.5_{-1.7}^{+1.7}$ & host cluster C1 1900+14 (Davies et al. 2009) \\
1RXS J170849.0-400910 & $3.8_{-0.5}^{+0.5}$ & red clump star method (Durant \& van Kerkwiik 2006) \\
1E 1048.1-5937 & $9.0_{-1.7}^{+1.7}$ & red clump star method (Durant \& van Kerkwijk 2006) \\
4U 0142+61 & $3.6_{-0.4}^{+0.4}$ & red clump star method (Durant \& van Kerkwiik 2006) \\
1E 2259+586 & $3.2_{-0.2}^{+0.2}$ & radio observation to SNR CTB 109 (Kothes et al. 2002) \\
\hline 1E 1547.0-5408 & $3.91_{-0.07}^{+0.07}$ & dust scattering halo (Tiengo et al. 2010) \\
SGR 0501+4516 & 3.3 & assuming on the Perseus arm \\
SGR 1833-0832 & 9.0 & assuming on the Scutum-Crux arm \\
CXOU J164710.2-455216 & $3.9_{-0.7}^{+0.7}$ & assocation to Westerlund I (Kothes \& Dougherty 2007) \\
Swift J1834.9-0846 & $4.2_{-0.3}^{+0.3}$ & association to SNR W41 (Leahy \& Tian 2008) \\
Swift J1822.3-1606 & $1.6_{-0.3}^{+0.3}$ & association to H Hi region M17 (Nielbock et al. 2001) \\
\hline
\end{tabular}

Note. - Referring to up-to-date data from Olausen \& Kaspi (2014) with previous distance studies: 1E 1841-045, >5 kpc (a red clump star method, Durant \& van Kerkwijk 2006); 1RXS J170849.0-400910, $3.2-4.0 \mathrm{kpc}$ (dust scattering halo, Rivera-Ingraham \& van Kerkwij] 2010); 1E 1048.1-5937, 5.7-6.2 kpc (dust scattering halo Rivera-Ingraham \& van Kerkwijk 2010); 4U 0142+61, 3.5-6.8 kpc (dust scattering halo, Rivera-Ingraham \& van Kerkwiik 2010); $1 \mathrm{E} 2259+586,3.0 \pm 0.5 \mathrm{kpc}$ (radio observation to SNR CTB 109 , Kothes et al. 2002), 7.5 \pm 1.0 (Red clump star method, Durant \& van Kerkwijk 2006), $4.0 \pm 0.8$ (radio observation to SNR CTB 109, Tian et al. 2010); 1E 1547.0-5408, $\sim 9 \mathrm{kpc}$ (diparsion measure, Camilo et al. 2007), $\sim 4 \mathrm{kpc}$ (possible association with SNR G327.24-0.13, Gelfand \& Gaensler 2007); SGR 0501+4516 $0.8 \pm 0.4 \mathrm{kpc}$ (possible association to SNR HB9, Leahy \& Tian 2007), 1.5 kpc (possible association to SNR HB9, Gaensler \& Chatterjee 2008), $2 \mathrm{kpc}$ (Lin et al.|2011); Swift J1834.9-0846, 5.4 kpc (dust scattering halo, Esposito et al. (2013a));

\section{DISTANCES}

In the present paper, we employed distances listed in Table 7, and illustrate their locations in Figure 17. For three sources, SGR 0501+4516, SGR 0419+5729, and SGR 1833-0832, which do not have any reliable distance measurement, we assumed that sources are on the Galactic spiral arms. We attribute SGR $0501+4516$ and SGR $0419+5729$ to the Perseus arm. We assume SGR 1833-0832 on the Scutum-Crux arm, since SGR 1833-0832 shows a similar high X-ray extinction $N_{\mathrm{H}}$ as that of SGR 1806-20 toward the inner Galaxy. It should be noted that the present distance are still highly uncertain, e.g., even for the well-studied object 1E 2259+586 hosted by SNR CTB 109, the distance has been revised several times within a range by a factor of a few (see note in Table $7 d \sim 3.0-7.5 \mathrm{kpc}$ for 1E 2259+586).

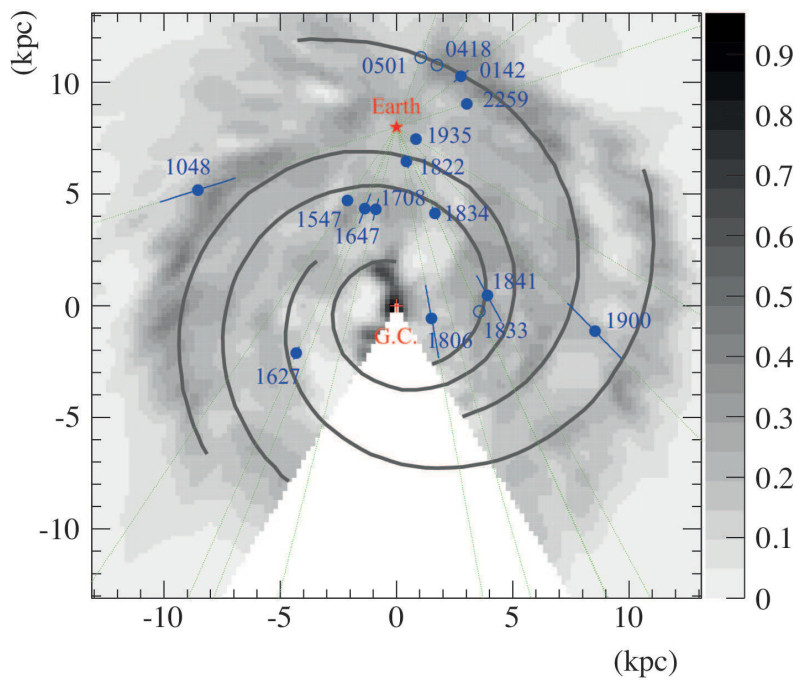

Figure 17. Galactic locations of SGRs and AXPs used in the present paper. The background plot indicates the atomic hydrogen $\left(H_{\mathrm{I}}\right)$ density map at the Galactic plane measured from a radio 21-cm line survey (Nakanishi \& Sofue 2003). The filled circles are objects with distance measurements (Table 7), while open symbols are sources without known distances and to be assumed on the Galactic spiral arms (Nakanishi \& Sofue 2006). 



Figure 18. Examples of the SXC decays of SGR 0501+4516 (left), Swift J1822-16069 (center), and SGR 0418+5729 (right). Top and bottom figures are the absorbed and unabsorbed $2-10 \mathrm{keV}$ X-ray fluxes. Fittings are performed with a single power-law (PL) model (gray dashed lines), a constant with an exponential mode (green dashed lines), a broken power-law model (blue dashed lines), and a plateau decay models (red thick lines). Ratio of data to models are shown in bottom panels of individual figures.

\section{DECAY FUNCTION}

Figure 18 is fitting examples of X-ray light curves during three outbursts which showed the plateau-like feature at their early phase. We tested four empirical formulae generally used in literatures: absorbed or unabsorbed fluxes $F(t)$ as a function of an elapsed time $t$ from onsets of outbursts are represented by 1 ) a single power-law $F(t)=K_{\mathrm{pl}} \cdot t^{-\alpha}$ where $\alpha$ and $K_{\mathrm{pl}}\left(\mathrm{erg} \mathrm{s}^{-1} \mathrm{~cm}^{-2}\right)$ are a slope and normalization, 2) an exponential shape $F(t)=F_{\text {const }}+K_{\exp } \cdot \exp \left(-t / \tau_{\exp }\right)$ where $\tau_{\exp }$ (days), $K_{\exp }\left(\mathrm{ergs}^{-1} \mathrm{~cm}^{-2}\right)$, and $F_{\text {const }}\left(\operatorname{erg~s}^{-1} \mathrm{~cm}^{-2}\right)$ are a decay time-scale, normalization, and constant, 3) a broken power-law $F(t)=K_{\text {brkn }} \cdot t^{-\beta_{1}}$ for $t<t_{\text {bread }}$, while $F(t)=K_{\text {brkn }} t_{\text {break }}^{-\left(\beta_{1}-\beta_{2}\right)} t^{-\beta_{2}}$ for $t \geq t_{\text {bread }}$, where $K_{\text {brkn }}$, $t_{\text {break }}, \beta_{1}$, and $\beta_{2}$ are a normalization, breaking time of the curve, and slopes before/after this break, and 4) the plateau decay (FD) function defined in Eq. (12). The PD model (4) generally gives good approximation of the light curves even though $\chi^{2}$ is close to that of the broken power-law case. For example, the $\chi^{2}$ (dof) of the absorbed flux of SGR 0418+5729 are 44.4 (27), 24.7 (27), 8.0 (25), and 8.3 (26) for the above 1), 2), 3) and 4) models, respectively.

The onset of light curves is fixed at the first short burst detected by Swift/BAT. Since we do not know the exact onset time of the X-ray outbursts, this might artificially produce the plateau like structure. Such a possibility was already discussed to explain a plateau phase of the X-ray afterglow of gamma-ray bursts (Yamazaki 2009). If $\tau_{0}$ is an artificial lag of the onset time, the single power-law is expanded to past typically around $t \sim-\tau_{0}$. Predicted X-ray flux at $t \sim-\tau_{0}$ becomes enough bright for the Monitor of All-sky X-ray Image (MAXI) (Matsuoka et al. 2009) to detect it. However the MAXI did not show strong enhancement (e.g., $7 \sigma$ upper-limit at $\sim 5$ mCrab level) for SGR 0418+5729, SGR 1833-0832, and Swift J1834.9-0846 (private communication with T. Mihara). Therefore, the plateau-decaying shape is not the artifact of the choice of the time origin. 